\title{
Unseen Artificial Intelligence-Deep Learning Paradigm for Segmentation of Low Atherosclerotic Plaque in Carotid Ultrasound: A Multicenter Cardiovascular Study
}

Pankaj K. Jain ${ }^{1}{ }^{\oplus}$, Neeraj Sharma ${ }^{1}$, Luca Saba ${ }^{2}$, Kosmas I. Paraskevas ${ }^{3}$, Mandeep K. Kalra ${ }^{4}$, Amer Johri ${ }^{5}$, John R. Laird ${ }^{6}$, Andrew N. Nicolaides ${ }^{7}$ and Jasjit S. Suri ${ }^{8, *,+}$

1 School of Biomedical Engineering, IIT (BHU), Varanasi 221005, India; pankajkrjain.rs.bme17@iitbhu.ac.in (P.K.J.); neeraj.bme@iitbhu.ac.in (N.S.)

2 Department of Radiology, Azienda Ospedaliero Universitaria (A.O.U.), 10015 Cagliari, Italy; lucasabamd@gmail.com

3 Department of Vascular Surgery, Central Clinic of Athens, 14122 Athens, Greece; paraskevask@hotmail.com

4 Department of Radiology, Massachusetts General Hospital, 55 Fruit Street, Boston, MA 02114, USA; MKALRA@mgh.harvard.edu

5 Division of Cardiology, Queen's University, Kingston, ON K7L 3N6, Canada; amerjohri@gmail.com

6 Heart and Vascular Institute, Adventist Health St. Helena, St. Helena, CA 94574, USA; Lairdjr@ah.org

check for updates

Citation: Jain, P.K.; Sharma, N.; Saba, L.; Paraskevas, K.I.; Kalra, M.K.; Johri, A.; Laird, J.R.; Nicolaides, A.N.; Suri,

J.S. Unseen Artificial Intelligence-Deep Learning Paradigm for Segmentation of Low Atherosclerotic Plaque in Carotid Ultrasound: A Multicenter Cardiovascular Study. Diagnostics 2021, 11, 2257. https://doi.org/ $10.3390 /$ diagnostics 11122257

\section{Academic Editors: Andreas}

Hjelm Brandt and Kristoffer Lindskov Hansen

Received: 8 November 2021

Accepted: 30 November 2021

Published: 2 December 2021

Publisher's Note: MDPI stays neutral with regard to jurisdictional claims in published maps and institutional affiliations.

Copyright: (c) 2021 by the authors. Licensee MDPI, Basel, Switzerland. This article is an open access article distributed under the terms and conditions of the Creative Commons Attribution (CC BY) license (https:/ / creativecommons.org/licenses/by/ $4.0 /)$.
7 Vascular Screening and Diagnostic Centre, University of Nicosia, Nicosia 1700, Cyprus; anicolaides1@gmail.com

8 Stroke Diagnostic and Monitoring Division, AtheroPoint ${ }^{\mathrm{TM}}$, Roseville, CA 95661, USA

* Correspondence: jasjit.suri@atheropoint.com; Tel.: +1-(916)-749-5628

+ Author: Jasjit S. Suri, FIEEE ${ }^{a}$, FAIMBE $^{b}$, FAIUM $^{c}$, FSVM $^{d}$, FAPVS ${ }^{\text {e }}$. ${ }^{a}$ Fellow, Institute of Electrical and Electronics Engineering; ${ }^{b}$ Fellow, American Institute of Medical and Biological Engineering; ${ }^{c}$ Fellow, American Institute of Ultrasound in Medicine; ${ }^{\mathrm{d}}$ Fellow, Society of Vascular Medicine; ${ }^{\mathrm{e}}$ Fellow, Asia Pacific Vascular Society.

Abstract: Background: The early detection of carotid wall plaque is recommended in the prevention of cardiovascular disease (CVD) in moderate-risk patients. Previous techniques for B-mode carotid atherosclerotic wall plaque segmentation used artificial intelligence (AI) methods on monoethnic databases, where training and testing are from the "same" ethnic group ("Seen AI"). Therefore, the versatility of the system is questionable. This is the first study of its kind that uses the "Unseen $\mathrm{AI}^{\prime}$ paradigm where training and testing are from "different" ethnic groups. We hypothesized that deep learning (DL) models should perform in 10\% proximity between "Unseen AI" and "Seen AI". Methodology: Two cohorts from multi-ethnic groups (330 Japanese and 300 Hong Kong (HK)) were used for the validation of our hypothesis. We used a four-layered UNet architecture for the segmentation of the atherosclerotic wall with low plaque. "Unseen AI" (training: Japanese,

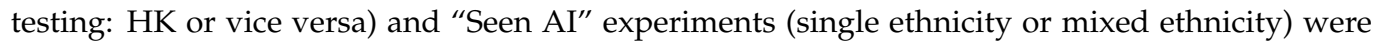
performed. Evaluation was conducted by measuring the wall plaque area. Statistical tests were conducted for its stability and reliability. Results: When using the UNet DL architecture, the "Unseen AI" pair one (Training: 330 Japanese and Testing: $300 \mathrm{HK}$ ), the mean accuracy, dicesimilarity, and correlation-coefficient were 98.55, 78.38, and $0.80(p<0.0001)$, respectively, while for “Unseen $\mathrm{AI}^{\prime}$ " pair two (Training: $300 \mathrm{HK}$ and Testing: 330 Japanese), these were 98.67, 82.49, and $0.87(p<0.0001)$, respectively. Using "Seen $\mathrm{AI}^{\prime}$, the same parameters were 99.01, 86.89 and 0.92 $(p<0.0001)$, respectively. Conclusion: We demonstrated that "Unseen AI" was in close proximity $(<10 \%)$ to "Seen $\mathrm{AI}^{\prime}$, validating our DL model for low atherosclerotic wall plaque segmentation. The online system runs $<1 \mathrm{~s}$.

Keywords: Unseen AI; Seen AI; UNet deep learning; multi-ethnic studies; carotid atherosclerotic wall plaque 


\section{Introduction}

\subsection{Stroke Statistics, Causes of Stroke, and Need for Screening}

Stroke is the third leading cause of death in the modern age. As per the 2018 data from the Centre for Disease Control and Prevention (CDC), one in every six deaths from cardiovascular diseases (CVD) is from stroke [1]. In the USA, every $40 \mathrm{~s}$, someone suffers from a stroke, and every $4 \mathrm{~min}$, someone dies of stroke [1]. The primary cause of stroke is the formation of atherosclerosis disease in carotid arteries [2], where the plaque is formed in the lumen-intima and media layers [3]. The LDL penetration in the arterial walls accelerate the plaque formation, such as fibrosis, fibrin, and macrophages due to a sedentary lifestyle [3]. This plaque then ruptures over time, causing embolism in the brain leading to stroke [2,4]. The plaque formation worsens with comorbidities such as diabetes [5], hypertension [6], renal disease [7], and heart disease [8]. Thus, it is vital to detect wall plaque during the early stages of its formation using angiography screening techniques [9-11].

\subsection{Importance of Imaging Modalities and Plaque Quantification}

Various imaging modalities such as magnetic resonance imaging (MRI) [9], computed tomography (CT) [12], and ultrasound (US) $[5,13]$ are used for the screening of the disease. The US is cheap, user-friendly, has a smaller foot print, is non-invasive, and does not use a radiation-based method [14]. Therefore, B-mode ultrasound scans are primarily used for the detection of atherosclerotic plaque in carotid arteries. These ultrasonic scans offer image-based phenotypes as leading biomarkers for stroke risk assessment such as carotid intima-media thickness (cIMT) [15], intima-media thickness variability (IMTV) [16,17], maximum plaque height (MPH) [18], total plaque area (TPA) [19-21], total plaque volume (TPV), and lumen diameter (LD) [22-24]. Various automated and semi-automated methods have been proposed to calculate imaging biomarkers in the past [25]. These biomarkers have their own relevance based on the study objective involved [26]. The measurement of the plaque area was proposed as an alternate method of stroke risk prediction [19]. The carotid plaque area could be manually delineated between lumen-intima (LI) and media-adventitia (MA) borders, but this is tedious, error-prone, time-consuming, and leads to inter-observer variabilities $[27,28]$. Thus, one needs to automatically estimate the plaque wall area $[29,30]$. All the above methods involve conventional and statistical image processing methods for the LI and MA borders' segmentation and quantification.

\subsection{Brief Background of AI Literature}

Artificial intelligence (AI)-based methods are becoming more popular in the healthcare industry for automated diagnosis and prognosis [31,32]. Using the AI methods, researchers contributed some ad hoc methods involving machine learning methods for stroke risk assessment. Suri and his group significantly contributed to research in stroke risk assessment using machine learning [33-36]. These machine learning methods are not entirely automated as these involve human intervention for the extraction of plaque features from the region of interest (ROI) area.

Deep learning (DL) techniques which are also part of AI have become more popular in healthcare imaging in recent years [37-42]. Deep learning techniques involve less human intervention and rely on directly extracting features from the images [43]. Several studies have been attempted recently using DL in medical imaging [44-47]. However, the current DL models use training and testing databases from the same ethnic group or cohort in recent studies. The database from one ethnic group or US scanner was is partitioned into training and testing pools. Therefore, the system only learns from the images coming from one ethnic group or US scanners. Thus, in a macro view, there are chances that the system is biased towards the images from one ethnic group or US scanner [48]. This view lays the foundation of the hypothesis of our research work. A few studies involve assessing CVD risk, but their focus was solely on enhancing the data size rather than studying the system's performance using a multi-ethnic database [49,50]. 


\subsection{Motivation, Hypothesis of Unseen AI, and Concept of Global Segmentation System}

We hypothesized that the DL model will be unbiased when we use separate training and testing databases. This means when we train the system with one ethnic group or cohort, it will be treated as a Unseen AI model for other ethnic groups or cohorts and vice versa. Therefore, we proposed two Unseen AI models which involve training the Japanese (Jap) cohort and testing on Hong Kong (HK) data and vice versa. We compared our Unseen AI model with the Seen AI model to validate our hypothesis that the Unseen AI systems are performing their function. We combined two ethnic data and made a pool of mixed data. This mixed database was used as the input to the Seen AI model. In the Seen AI model, we cross-validated each image using 10-fold cross-validations. Thus, in the Seen AI model, the cohort type was known to the system. Furthermore, we can also check the performance of the Unseen AI model against the Seen AI model by cross-validating each cohort. Figure 1 shows the global system diagram for unseen image segmentation.

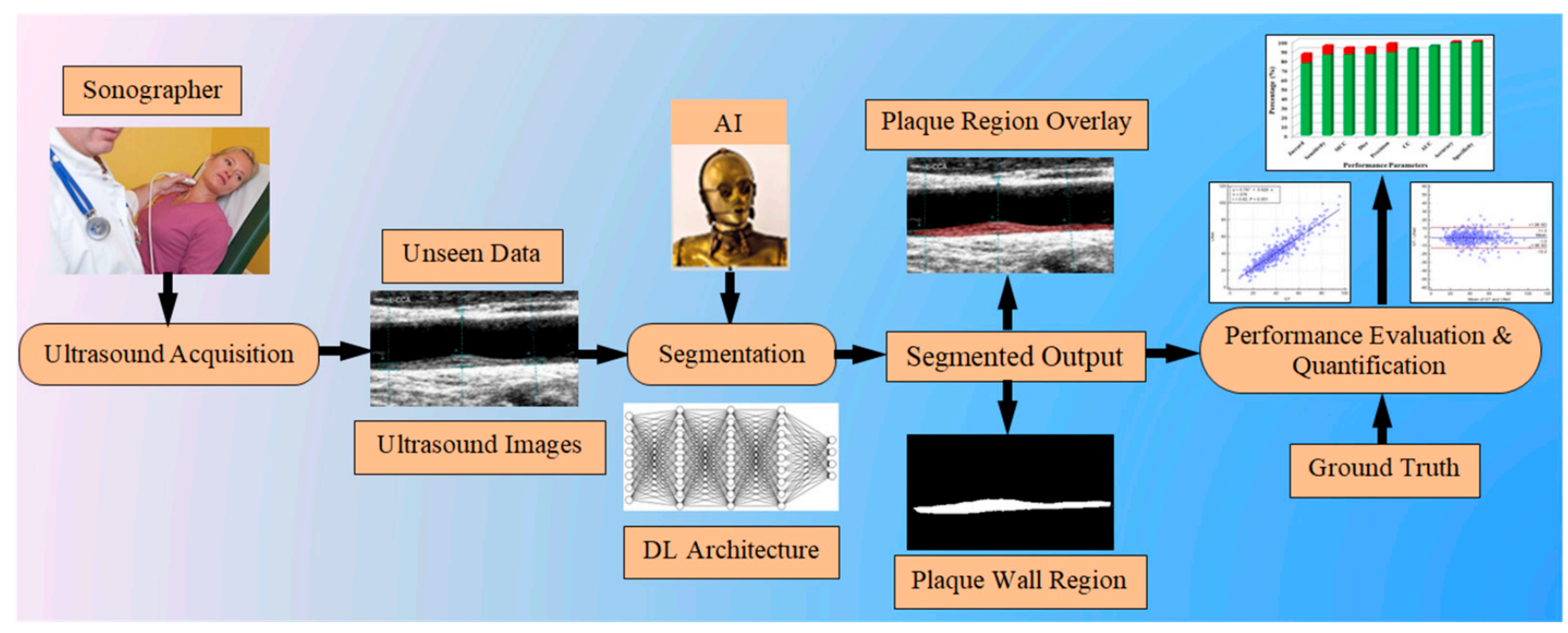

Figure 1. System diagram for unseen data processing.

\subsection{Layout of This Study}

The architecture of this article includes six major sections. Section 1 describes the introduction, background, and hypothesis of the current work. The methodology section consists of patient's demographics, data collection and ground-truth data preparation, UNet-based deep learning architecture, and experimental protocols. All these sub-sections are described in Section 2. Section 3 contains the results of all experiments described in the methodology section. Section 4 describes the performance evaluation section where each performance tests results and their corresponding graphs are described. Finally, Sections 5 and 6 contain the discussion and conclusion.

\section{Methodology}

To prove our hypothesis via our experiments, the choice of the deep learning architecture and the image dataset is essential. We took special care in the architecture design and the multi-ethnic datasets, which were vital for the "Unseen AI" analysis and benchmarking against "Seen AI" standard protocols. Thus, the methodology section mainly consists of three parts: (1) patient demographics, data collection, and data preparation for multi-ethnic datasets; (2) DL architecture; and (3) the experimental protocol used for the "Unseen $\mathrm{AI}^{\text {" }}$

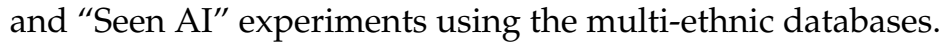




\subsection{Patient Demographics, Data Collection, and Data Preparation}

\subsubsection{Patient Demographics for the First Group: Japanese Cohort}

The Japanese cohort consisted of 330 of left and right common carotid arteries Bmode ultrasound images taken from 165 patients. The male/female proportion in the cohort was $127 / 38$. All patients were examined retrospectively, and ethics approval was granted by the institutional review board (IRB) of Toho University, Japan. All patients were informed, and written consent was obtained before the examinations. The baseline characteristics of the Japanese diabetes patients included a mean age of $68.25 \pm 11.23$ years. The mean hemoglobin (HbA1c) of all patients was $6.22 \pm 1.04 \mathrm{mg} / \mathrm{dL}$, low density lipoprotein (LDL) cholesterol of $101.59 \pm 31.03(\mathrm{mg} / \mathrm{dL})$, high density lipoprotein (HDL) cholesterol of $51.05 \pm 14.56(\mathrm{mg} / \mathrm{dL})$, and total cholesterol (TC) of $175.39 \pm 36.03(\mathrm{mg} / \mathrm{dL})$. Out of 165 patients, 73 patients were smokers, 19 had a family history of CVD and 116 had hypertension. The mean systolic and diastolic blood pressure values were $134.06 \pm 9.14(\mathrm{~mm} / \mathrm{Hg})$ and $87.03 \pm 4.57(\mathrm{~mm} / \mathrm{Hg})$. The mean eGFR value for the cohort was $47.31 \pm 19.93\left(\mathrm{~mL} / \mathrm{min} / 1.73 \mathrm{~m}^{2}\right)$. This dataset was used in our previous studies $[33,34,40]$.

\subsubsection{Patient Demographics for the Second Group: Hong Kong Cohort}

A total of 300 images from 50 patients (6 images per patient, 3 scans at each side of neck showing three different acquisition scans: anterior, anterolateral, and posterolateral with simultaneous ECG gating). All subjects were postmenopausal Chinese women aged between 54 and 67 years (mean age 60.2 years). Subjects were informed and consent was obtained before their inclusion in study. In the pool of 50 females, 28 females were diagnosed with different diseases: one was diagnosed with diabetes, three with hypertension, seven were diagnosed with hypercholesterolemia, whereas 15 had both hypertension and hypercholesterolemia, and two had all three abnormalities. The rest controlled population of 22 females had normal blood pressure, total cholesterol, and glucose levels in fasting blood. These data were used in one of our previous studies [15].

\subsubsection{Data Acquisition and Ultrasound Imaging for the Two Ethnic Groups}

Japanese Cohort: All Japanese ultrasound images were scanned by Aplio XV, Aplio XG, Xario, Toshiba, Inc., Tokyo, Japan Ultrasound scanner, equipped with a 7.5 MHz linear array transducer. An experienced sonographer performed all US scans. The timeline of data collection was between July 2009 and September 2010. The recommendations of the American Society of Echocardiography Carotid Intima-Media Thickness Task Force were followed during the acquisition of these images. In this database, the mean resolution was $0.052 \pm 0.01 \mathrm{~mm} /$ pixel.

Hong Kong Cohort: The Hong Kong (HK) database was examined using Sonoline Antares (Siemens Medical Solutions, USA, Inc., Malvarn, PA, USA) ultrasound scanner equipped with a $13.5 \mathrm{MHz}$ linear transducer. The sonographer digitally captured a $10 \mathrm{~s}$ clip of each scan for offline analysis. Similarly, six segments (3 left and 3 right) of the left and right carotid arteries were archived for each subject. These data were used in some of our previous studies $[15,51]$.

\subsubsection{Ground-Truth Data Preparation}

An image-processing expert trained by an experienced cardiologist prepared the ground-truth binary mask images. We used a clinically acceptable image tracing tool developed by Atheropoint ${ }^{\mathrm{TM}}$ LLC, Roseville, CA, USA, to trace the CCA images' lumenintima (LI) and media-adventitia (MA) borders. The region between the lumen-intima (LI) and media-adventitia (MA), i.e., the atherosclerotic wall plaque, generates binary masks for the DL system. We used PowerToys (Microsoft) software for resizing the raw images to a size of $224 \times 224 \times 3$. Additionally, the corresponding binary mask was also resized to the same size as of raw images using same software. 


\subsection{UNet-Based Deep Learning Architecture}

A four-layer DL architecture UNet with a stack of four encoders and four decoder stages on both sides of the U-shape is shown in Figure 2. The encoder stages up-sample the images, while the decoder stages down-sample the images. Each encoder stage of UNet consists of a 2D-convolution layer (red) followed by ReLU (turquoise) and a MaxPooling layer (yellow). Similarly, each decoder stage consists of a stack of up-convolution-2D layers (dark green), depth-concatenation (light green), 2D-convolution (red) ReLU (turquoise) and a MaxPooling layer (yellow). A grayscale US image was given as an input of size $224 \times 224$ from the encoder stage 1 . At stage one, the number of convolution filters was 64 , which doubled in each next stage of the encoder module. Therefore, the numbers of filters in each stage become 128, 256, and 512. In contrast, the number of filters is reduced to half in each stage of the decoder. When counting from the bottom of the picture, these numbers are $512,256,128$, and 64 . Both the encoder and decoder modules are connected via a bridge network. The bridge network consists of $3 \times 3 \times 1024$ filters. The bridge network provides a stack of features that are compatible with concatenating to the last encoder layer after downsampling from the first upsampling layer.

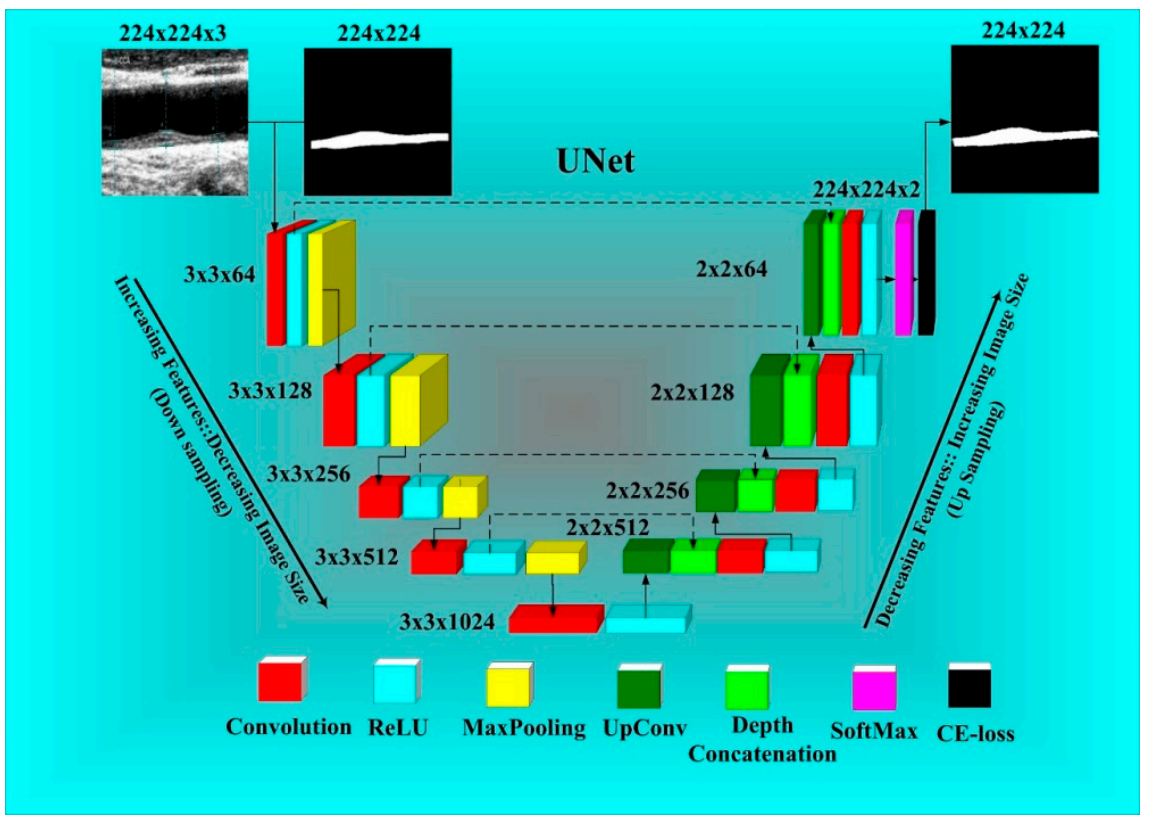

Figure 2. UNet: Four-layer DL architecture for atherosclerotic plaque wall segmentation.

From each encoder stage, spatial features are extracted and transferred to the downsampling layer at the corresponding level of depth via a skip connection. Furthermore, these features are added with the features of the previous decoder or bridge network layers. Finally, after the last decoder stage, the image features are classified into two classes, i.e., the plaque area and the background using the softmax classifier layer (pink). An efficient ADAM optimizer was used to reduce the cross-entropy loss in plaque segmentation. If $y_{i}$ represents the GT label and $a_{i}$ means the softmax classifier probability, then the cross-entropy $(C E)$ loss is described by Equation (1) as follows:

$$
L_{C E}=-\left[\left(\mathrm{y}_{\mathrm{i}} \times \log \mathrm{a}_{\mathrm{i}}\right)+\left(1-\mathrm{y}_{\mathrm{i}}\right) \times \log \left(1-\mathrm{a}_{\mathrm{i}}\right)\right]
$$

\subsection{Experimental Protocol}

We performed various experiments using both the Japanese and Hong Kong databases. Table 1 represents a consolidated list of the experiments with selected combinations of the databases. All experiments were conducted in the same programming environment (MATLAB 2019b) as well as with the same hardware configuration (core i7 8th Gen, 16 GB 
RAM, 8 GB NVIDIA Quadro P4000 Graphics processor) and operating system using Windows 10. Additionally, the same hyperparameters were applied to all experiments such as: batch size $=10$, epochs $=100$, optimizer $=\mathrm{ADAM}$, learning rate $=1 \times 10^{-4}$ Input grayscale image and binary mask image sizes were also fixed to $224 \times 224$ for all experiments.

Table 1. Summary of all experiments performed on a multiethnic database.

\begin{tabular}{ccccc}
\hline Exp \# & Name of Exp & Training DB & Testing DB & $\begin{array}{c}\text { Training } \\
\text { Protocol }\end{array}$ \\
\hline Exp \#1 & $\begin{array}{c}\text { Unseen AI-1 (Tr: JAP, Te: } \\
\text { HK) }\end{array}$ & Japanese 330 & Hong Kong 300 & $\begin{array}{c}\text { All Japanese DB } \\
\text { for training }\end{array}$ \\
\hline Exp \#2 & $\begin{array}{c}\text { Unseen AI-2 (Tr: HK, Te: } \\
\text { JAP) }\end{array}$ & Hong Kong 300 & Japanese 330 & $\begin{array}{c}\text { All Hong Kong } \\
\text { DB for training }\end{array}$ \\
\hline Exp \#3 & Seen AI-1; CV w / Mixed & $\begin{array}{c}\text { Japanese (330) + } \\
\text { Hong Kong (250) }\end{array}$ & $\begin{array}{c}\text { Japanese (330) + } \\
\text { Hong Kong (250) }\end{array}$ & $\begin{array}{c}\text { 10-fold } \\
\text { cross-validation }\end{array}$ \\
\hline Exp \#4 & Seen AI-2; CV w / JAP & Japanese (330) & Japanese (330) & $\begin{array}{c}\text { 10-fold } \\
\text { cross-validation }\end{array}$ \\
\hline Exp \#5 & Seen AI-3; CV w / HK & Hong Kong (300) & Hong Kong (300) & $\begin{array}{c}\text { 10-fold } \\
\text { cross-validation }\end{array}$ \\
\hline CV w/: cross-validation with; JAP: Japanese; HK: Hong Kong. & &
\end{tabular}

\subsubsection{Unseen AI Data Experiments}

In experiment\#1, (Unseen AI-1 (Tr: JAP, Te: HK)), we trained the UNet model with 330 low-risk images from Japanese DB and the trained model was saved. Furthermore, we tested the model on 300 HK DB. During the experiment, an entire database of a single ethnic group was used for training and DB from other ethnic groups was used for testing. Experiment\#2 (Unseen AI-2 (Tr: HK, Te: JAP)) is the reverse of experiment\#1 (Unseen AI-1 (Tr: JAP, Te: HK)), in which we performed the training of the same network with $300 \mathrm{HK}$ databases and testing on 330 Japanese databases. In both experiments, one database was used for training and the other was used for testing.

\subsubsection{Seen AI Data Experiments}

Furthermore, we decided to check the same network with a mixture of both databases. Continuing with our hypothesis, in this experiment\#3 (Seen AI-1 CV w/ Mixed), we mixed up 330 Japanese and $300 \mathrm{HK}$ databases and used the 10-fold partition method to crossvalidate each image. In the 10 -fold cross-validation method, $90 \%$ of images of the mixed database were used as the training images and $10 \%$ of the images were used as the test images. Furthermore, $10 \%$ of the test images were swapped from the original database, and a fresh batch of $10 \%$ test images became available for testing. Likewise, 10 different sets of $10 \%$ images were used for testing; therefore, the experiment was repeated 10 times. Thus, in a 10-fold cross-validation, each image gets a chance to go for testing.

Alongside the mixed database, we also checked the performance of the UNet model for individual databases by only cross-validating a single database. In experiment\#4 (Seen AI-2 CV w/ JAP), we only used the Japanese DB and cross-validated all 330 images using the 10-fold cross-validations explained earlier. Similarly, in experiment \#5 (Seen AI-3 CV w/ HK)), we performed 10-fold cross-validations on the 300-image Hong Kong DB. Table 2 show the summary of the experiments used in this study. 
Table 2. Classification parameters of the test dataset of all experiments.

\begin{tabular}{|c|c|c|c|c|c|c|c|c|}
\hline Experiment \# & UNet Experiments & $\mathrm{ACC}$ & Sens & Spec & Prec & MCC & DSC & JI \\
\hline Exp \#1 & $\begin{array}{c}\text { Unseen AI-1 (Tr: JAP, Te: } \\
\text { HK) }\end{array}$ & $\begin{array}{c}98.55 \pm \\
0.57\end{array}$ & $\begin{array}{l}95.41 \pm \\
5.29\end{array}$ & $\begin{array}{c}98.64 \pm \\
0.62\end{array}$ & $\begin{array}{c}67.82 \pm \\
12.55\end{array}$ & $\begin{array}{l}79.29 \pm \\
8.64\end{array}$ & $\begin{array}{c}78.38 \pm \\
10.11\end{array}$ & $65.42 \pm 11.84$ \\
\hline Exp \#2 & $\begin{array}{c}\text { Unseen AI-2 (Tr: HK, Te: } \\
\text { JAP) }\end{array}$ & $\begin{array}{c}98.67 \pm \\
0.67\end{array}$ & $\begin{array}{c}79.52 \pm \\
8.84\end{array}$ & $\begin{array}{c}99.47 \pm \\
0.67\end{array}$ & $\begin{array}{c}87.29 \pm \\
12.45\end{array}$ & $\begin{array}{c}82.29 \pm \\
8.34\end{array}$ & $\begin{array}{c}82.49 \pm \\
8.44\end{array}$ & $70.98 \pm 10.90$ \\
\hline Exp \#3 & Seen AI-1 CV w/ Mixed & $\begin{array}{c}99.01 \pm \\
0.44\end{array}$ & $\begin{array}{c}86.37 \pm \\
8.69\end{array}$ & $\begin{array}{c}99.52 \pm \\
0.41\end{array}$ & $\begin{array}{c}88.55 \pm \\
8.82\end{array}$ & $\begin{array}{c}86.68 \pm \\
6.19\end{array}$ & $\begin{array}{c}86.89 \pm \\
6.43\end{array}$ & $77.34 \pm 9.15$ \\
\hline Exp \#4 & Seen AI-2 CV w/ JAP & $\begin{array}{c}98.99 \pm \\
0.58\end{array}$ & $\begin{array}{c}91.25 \pm \\
8.13\end{array}$ & $\begin{array}{c}99.26 \pm \\
0.64\end{array}$ & $\begin{array}{c}81.01 \pm \\
14.80\end{array}$ & $\begin{array}{c}84.88 \pm \\
9.49\end{array}$ & $\begin{array}{c}84.65 \pm \\
10.68\end{array}$ & $74.62 \pm 13.54$ \\
\hline Exp \#5 & Seen AI-3 CV w/ HK & $\begin{array}{c}98.96 \pm \\
0.39\end{array}$ & $\begin{array}{c}87.27 \pm \\
7.70\end{array}$ & $\begin{array}{c}99.43 \pm \\
0.42\end{array}$ & $\begin{array}{c}86.50 \pm \\
10.45\end{array}$ & $\begin{array}{c}86.04 \pm \\
7.72\end{array}$ & $\begin{array}{c}86.29 \pm \\
8.31\end{array}$ & $76.59 \pm 9.96$ \\
\hline
\end{tabular}

ACC: accuracy; Sens: sensitivity; Spec: specificity; Prec: precision; MCC: Mathew's correlation coefficient; DSC: dice similarity coefficient; JI: Jaccard index.

\section{Results}

In this section, we will discuss the classification results of all experiments listed in Table 2. Table 2 contains a summary of the classification results of all experiments. The classification parameters of the UNet training model include (i) the correlation coefficient (CC); (ii) the area under the curve (AUC); (iii) accuracy; (iv) sensitivity; (v) specificity; (vi) precision; (vii) Mathew's correlation coefficient; (viii) dice similarity coefficient (DSC); and (ix) the Jaccard index (JI). In the first experiment\#1, i.e., (Unseen AI-1 (Tr: JAP, Te: $\mathrm{HK})$ ), the UNet model was trained with 330 Japanese DB images and tested on 300 Hong Kong DB images. Therefore, nine mean classification parameters' values of the $300 \mathrm{HK}$ DB images are $0.8,0.87,98.55,95.41,98.64,67.82,79.29,78.38$, and 65.42 , respectively. Figure $3 a$ shows the bar chart of the mean classification parameters of the Unseen AI-1 (Tr: JAP, Te: HK). Now, in experiment\#2 (Unseen AI-2 (Tr: HK, Te: JAP)), the training and testing databases were swapped. The same model was trained on $300 \mathrm{HK}$ DB images and tested on 330 Japanese DB. Therefore, with Unseen AI-2 (Tr: HK, Te: JAP), we achieved nine mean classification parameters' values for the 330 Japanese DB images as 0.87, 0.94, 98.67, 79.52, $99.47,87.29,82.29,82.49$, and 70.98 . Figure $3 \mathrm{~b}$ shows the bar chart of the mean classification parameters of the Unseen AI-2 (Tr: HK, Te: JAP).

(a) Training: Japanese; Testing: Hong Kong

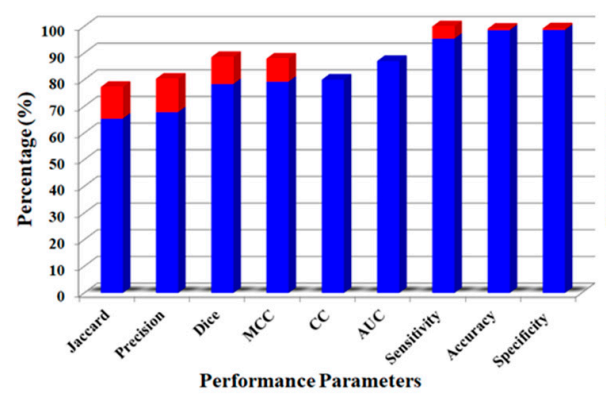

(b) Training: Hong Kong; Testing: Japanese

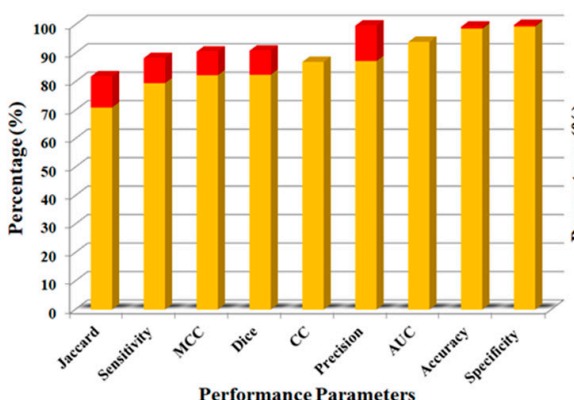

(c) $90 \%$ Train: $10 \%$ Test- Mix(Hong Kong + Japanese)

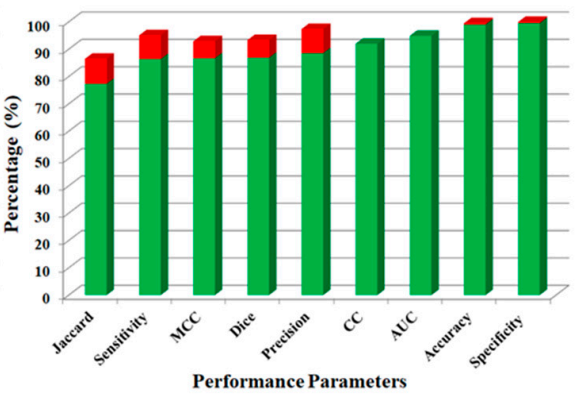

Figure 3. Bar chart for all performance parameters of Unseen AI and Seen AI experiments.

Now, in experiment \#3, i.e., (Seen AI-1 CV w / Mixed), the Japanese DB and Hong Kong DB was mixed and the combined DB was cross-validated using 10-fold cross-validations. Therefore, in this experiment using cross-validation, each image was tested once. The mean classification parameters' values for the mixed database were $0.92,0.95,99.01,86.37$, $99.52,88.55,86.68,86.89$, and 77.34. Figure $3 \mathrm{c}$ shows the bar chart of the mean classification parameters of experiment \#3, i.e., (Seen AI-1 CV w/ Mixed).

We also tested the monoethnic DB using the 10-fold cross-validation method. Using this strategy, we performed experiment\#4 (Seen AI-2 CV w/ JAP) in which only 330 
Japanese DB images were used for the 10-fold cross-validation. For this experiment, the mean classification parameters' values are $0.87,0.93,98.99,91.25,99.26,81.01,84.88,84.65$, and 74.62. In an experiment similar to experiment\#5 (Seen AI-3 CV w/ HK), only $300 \mathrm{HK}$ DB images were used for 10 -fold cross-validation. The mean classification parameters are $0.89,0.95,98.96,87.27,99.43,86.50,86.04,86.29$, and 76.59.

\section{Visual Segmentation Results}

Figure 4 below shows the generation of difference between and the GT images and AI segmented images. Figure 4a shows the overlay of the GT mask and raw image in green color. Figure $4 \mathrm{~b}$ shows the overlay of the AI-segmented mask and raw image in red color. Figure 4c shows the overlay of difference between the GT and AI segmented images. Thus, in Figure 4c, the red color shows the AI predicted plaque area whereas the green color represents the difference plaque area.

(a)
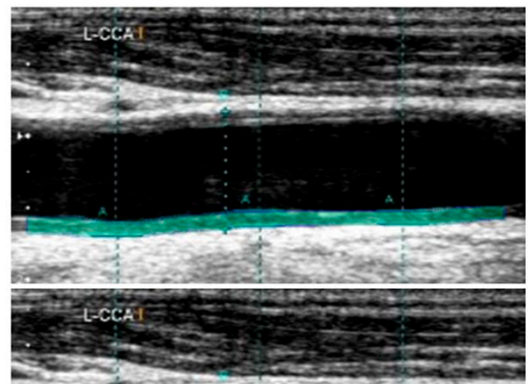

(b)

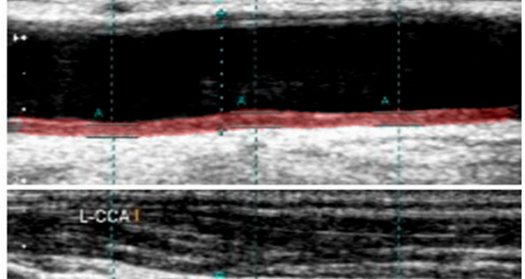

(c)

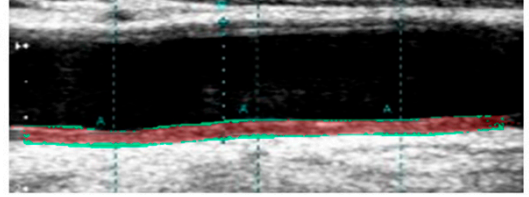

Figure 4. Generation of difference between the GT and AI plaque overlay images. (a) GT plaque overlay (b) AI-estimated plaque overlay (c) Difference between GT and AI plaque overlay image.

Figure 5 below shows the absolute difference image of the UNet model output (red) and GT mask overlay on the grayscale image (green). 
Unseen Exp1; Tr: Japanese (330); Te: Hong Kong (300)

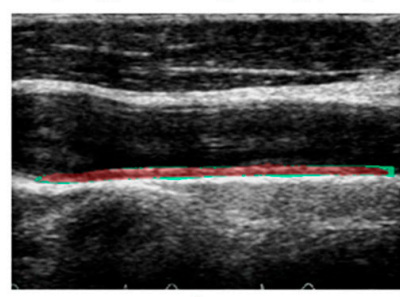

GTPA $=35.43 \mathrm{~mm}^{2}$ Est PA $=32.08 \mathrm{~mm}^{2}$

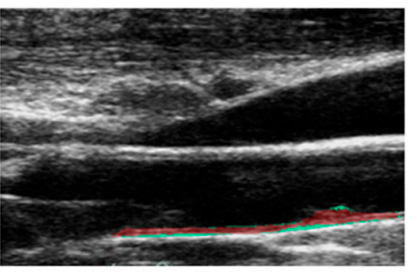

GTPA $=24.47 \mathrm{~mm}^{2}$ Est PA $=21.48 \mathrm{~mm}^{2}$

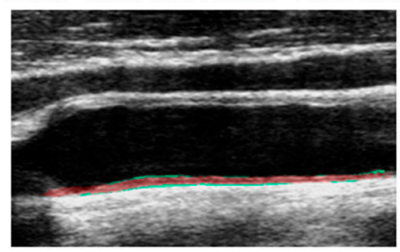

GTPA $=28.08 \mathrm{~mm}^{2}$ Est PA $=26.97 \mathrm{~mm}$
Unseen Exp2; Tr: Hong Kong

(300); Te: Japanese (330)

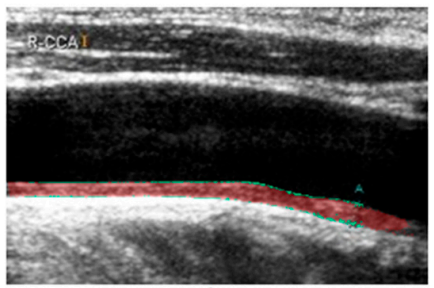

GTPA $=35.75 \mathrm{~mm}^{2}$ Est PA $=43.37 \mathrm{~mm}^{2}$

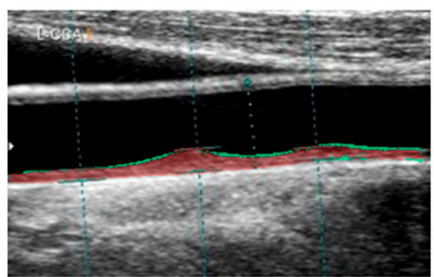

GTPA $=38.20 \mathrm{~mm}^{2}$ Est PA $=43.90 \mathrm{~mm}^{2}$

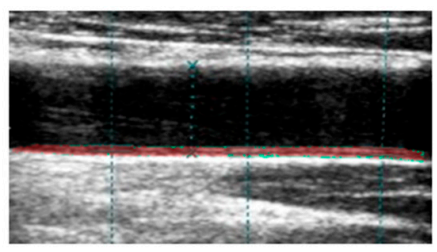

GTPA $=49.43 \mathrm{~mm}^{2}$ Est PA $=62.88 \mathrm{~mm}^{2}$
Seen Exp1; Tr+Te: Japanese (330) + Hong Kong (250)

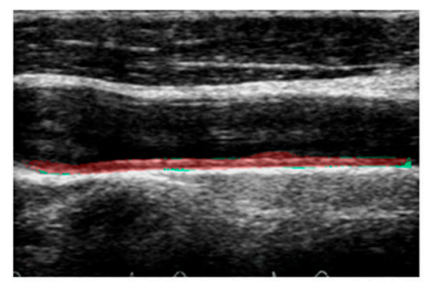

GTPA $=35.43 \mathrm{~mm}^{2} \mathrm{Est}$ PA $=40.09 \mathrm{~mm}^{2}$

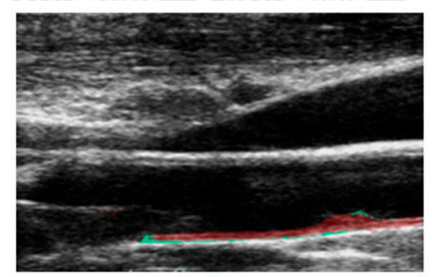

GTPA $=24.47 \mathrm{~mm}^{2}$ Est PA $=26.01 \mathrm{~mm}^{2}$

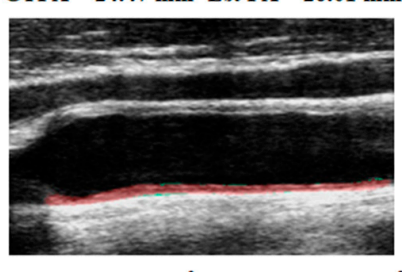

GTPA $=28.08 \mathrm{~mm}^{2}$ Est PA $=33.71 \mathrm{~mm}^{2}$

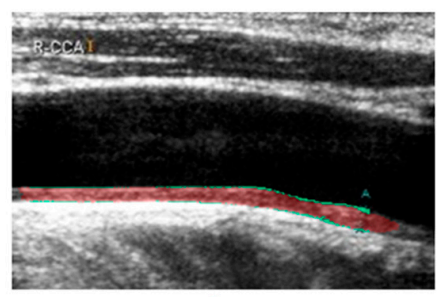

GTPA $=35.75 \mathrm{~mm}^{2}$ Est PA $=39.92 \mathrm{~mm}^{2}$

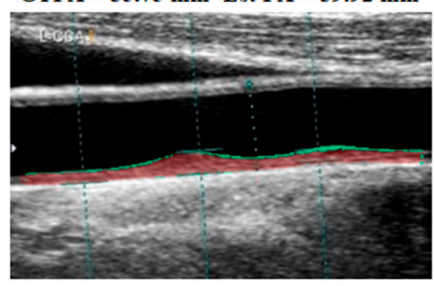

GTPA $=38.20 \mathrm{~mm}^{2}$ Est PA $=42.5 \mathrm{~mm}^{2}$

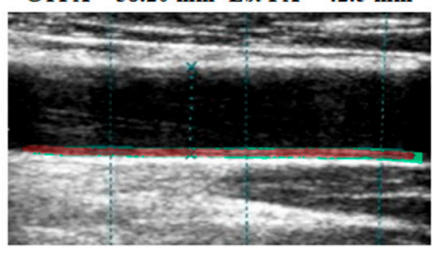

GTPA $=49.43 \mathrm{~mm}^{2}$ Est PA $=50.95 \mathrm{~mm}^{2}$

Figure 5. Visual results of the Unseen AI and Seen AI experiments. [Column 1 shows the absolute difference images of the HK test DB for experiment\#1 (Unseen AI-1 (Tr: JAP, Te: HK)). Similarly, Column 2 shows the absolute difference images of the Japanese test DB for experiment\#2 (Unseen AI-2 (Tr: HK, Te: JAP)). However, columns 3 and 4 represent the difference images from the mixed database of experiment\#3 (Seen AI-1 CV w/ Mixed)].

\section{Performance Evaluation}

We performed various performance evaluations and statistical tests on the test data to validate our hypothesis. We calculated the mean of all performance and statistical parameters for both DL models and presented them in Table 3.

Table 3. Performance and statistical parameters of the test dataset of all experiments.

\begin{tabular}{ccccc}
\hline Experiment \# & UNet Experiment & CC & AUC & FoM \\
\hline Exp \#1 & Unseen AI-1 (Tr: JAP, Te: HK) & 0.8 & 0.87 & 70.96 \\
\hline Exp\#2 & Unseen AI-2 (Tr: HK, Te: JAP) & 0.87 & 0.94 & 91.14 \\
\hline Exp \#3 & Seen AI-1, CV w/ Mixed & 0.92 & 0.95 & 97.57 \\
\hline Exp \#4 & Seen AI-2, CV w/ JAP & 0.87 & 0.93 & 88.89 \\
\hline Exp \#5 & Seen AI-3, CV w/ HK & 0.89 & 0.95 & 99.14 \\
\hline
\end{tabular}

CC: correlation coefficient; AUC: area under the curve; FoM: figure of merit.

\subsection{Correlation between AI Models and Ground Truth}

The correlation coefficient (CC) is an effective statistical parameter used to analyze the relationship between two quantities. It ranges between " 0 " and " 1 ", representing a degree of match between the two quantities. A high value (close to "1") represents a high match, whereas a low value represents a low match. Table 3 shows the CC value for all experiments. Additionally, Figure $6 \mathrm{a}-\mathrm{c}$ below show the $\mathrm{CC}$ values and regression curve for experiments \#1,\#2, and \#3. As depicted in the figure, the CC between UNet and GT for experiment \#1 (Unseen AI-1 (Tr: JAP, Te: HK)) is 0.8, 0.87 for experiment \#2 (Unseen AI-2 (Tr: HK, Te: JAP)), and 0.92 for experiment \#3 (Seen AI-1 CV w/ Mixed)—all having 
$p<0.001$. Additionally, the CC for exp\#4 (Seen AI-2 CV w/ JAP) is 0.87 , and 0.89 for experiment \#5 (Seen AI-3 CV w/ HK).

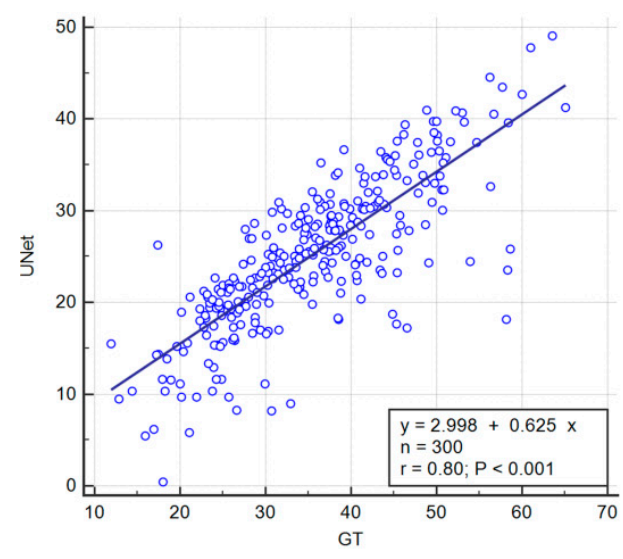

(a)

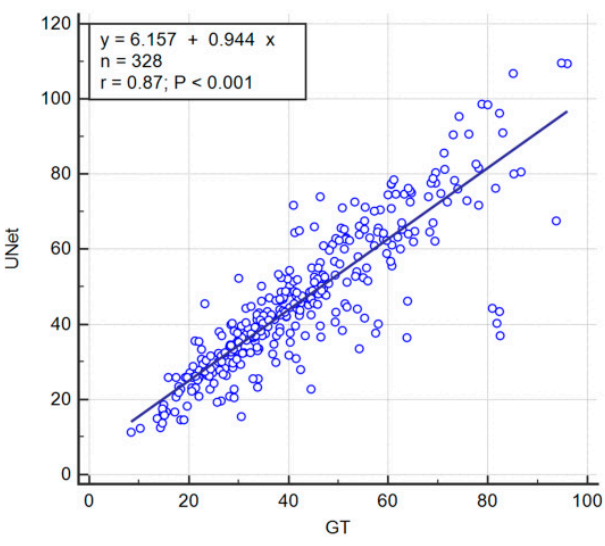

(b)

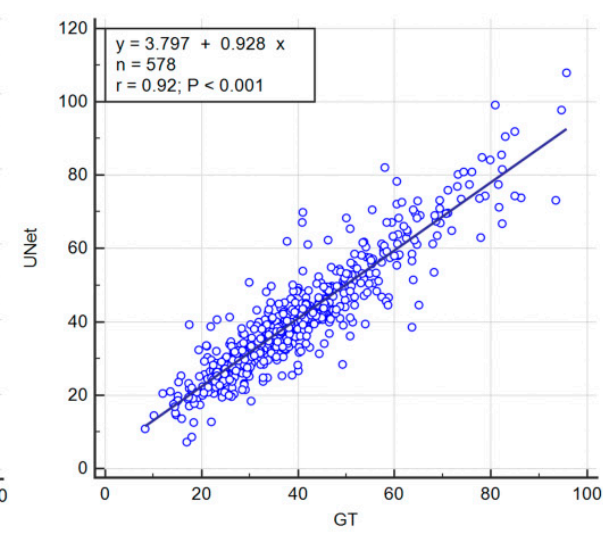

(c)

Figure 6. Regression plots for Unseen AI and Seen AI experiments. (a) Experiment \#1 (Unseen AI-1 (Tr: JAP, Te: HK)) (b) experiment \#2 (Unseen AI-2 (Tr: HK, Te: JAP)) (c) experiment \#3 (Seen AI-1 CV w/ Mixed).

\subsection{Receiver Operating Characteristics and AUC}

The receiver operating characteristics (ROC) curve and the area under the ROC curve are important performance parameters in medical image analysis. We used the groundtruth plaque area (GTPA) threshold value of $40 \mathrm{~mm}^{2}$ to generate the GT binary labels "1" for high-risk images and " 0 " for low-risk images. Furthermore, the deep learning plaque area (DLPA) was used to plot the ROC curve between the GTPA labels and DLPA scores. Figure 7a-c show the ROC curves and AUC values for experiment \#1 (Unseen AI-1 (Tr: JAP, Te: HK)), experiment \#2 (Unseen AI-2 (Tr: HK, Te: JAP)), and experiment \#3 (Seen AI-1 CV w/ Mixed).

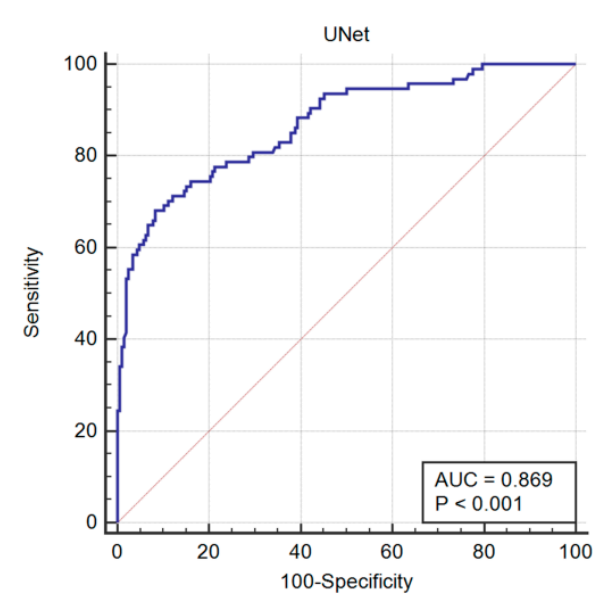

(a)

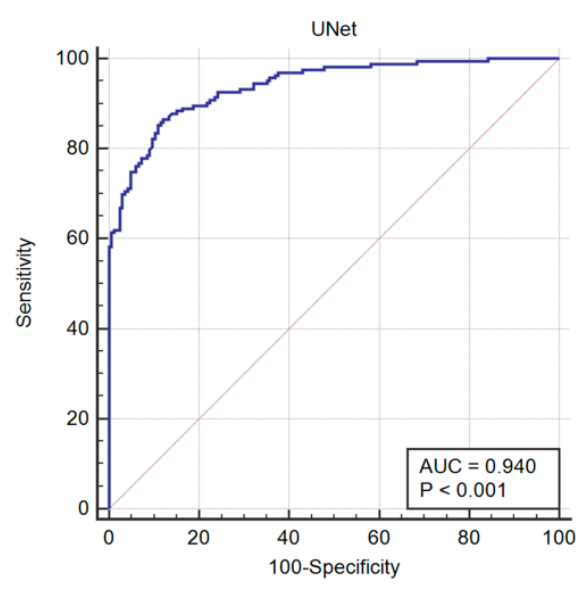

(b)

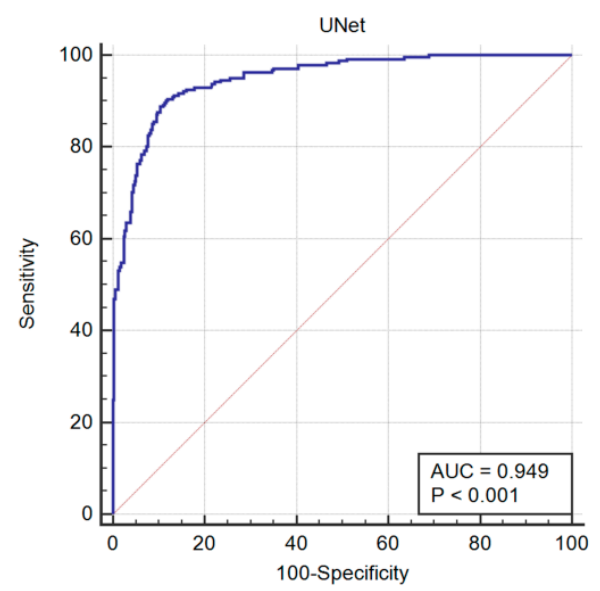

(c)

Figure 7. Receiver operating characteristics (ROC) curves and AUCs for the Unseen AI and Seen AI experiments. (a) Experiment \#1 (Unseen AI-1 (Tr: JAP, Te: HK)) (b) experiment \#2 (Unseen AI-2 (Tr: HK, Te: JAP)) (c) experiment \#3 (Seen AI-1 CV w/ Mixed).

\subsection{Bland-Altman Plots}

Bland-Altman's plots between the UNet-GT for experiment \#1 (Unseen AI-1 (Tr: JAP, Te: HK)), experiment \#2 (Unseen AI-2 (Tr: HK, Te: JAP)), and experiment \#3 (Seen AI-1 CV 
w/ Mixed) are shown in Figure 8a-c. From the plots, the Seen AI experiment \#1 model's output is closely concentrated along the central lines.

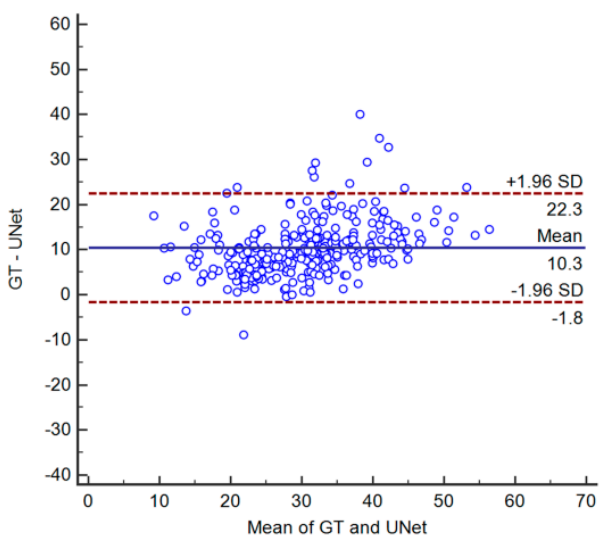

(a)

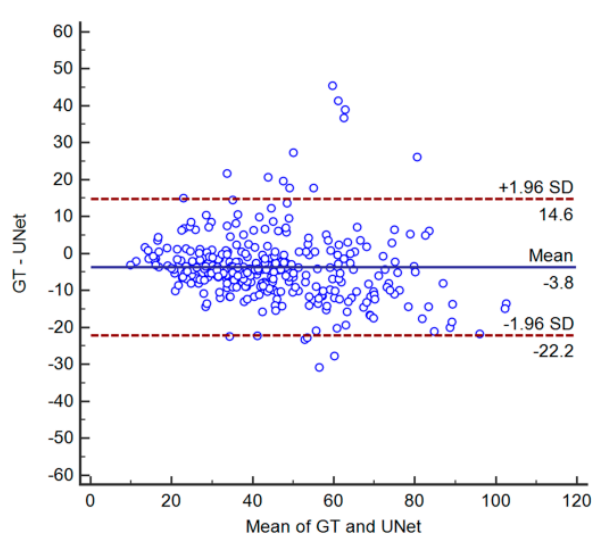

(b)

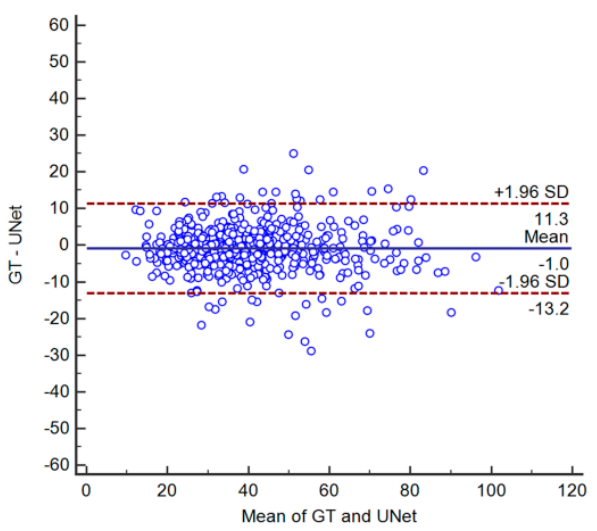

(c)

Figure 8. Bland-Altman's plots for Unseen and Seen experiments. (a) Experiment \#1 (Unseen AI-1 (Tr: JAP, Te: HK)) (b) experiment \#2 (Unseen AI-2 (Tr: HK, Te: JAP)) (c) experiment \#3 (Seen AI-1 CV w/ Mixed).

\subsection{Paired Sample t-Test and ANOVA Test}

Paired Sample $t$-test: The box and whiskers plot is a convenient way to show the data distribution. Figure $9 \mathrm{a}-\mathrm{c}$ below show the paired sample $t$-tests between the UNet estimated area and GT area using a box and whiskers plot for experiments \#1, 2, and 3, respectively. As the boxes in the plot show, for experiment \#3 (Seen AI-1 CV w/ Mixed), the median values of UNet are close to the GT median values compared to other experiments. Figure 10a-c show the box and whiskers plot for the analysis of variance (ANOVA) test between the UNet and GT area for all three experiments: experiment \#1 (Unseen AI-1 (Tr: JAP, Te: HK)), experiment \#2 (Unseen AI-2 (Tr: HK, Te: JAP)), and experiment \#3 (Seen AI-1 CV w/ Mixed).

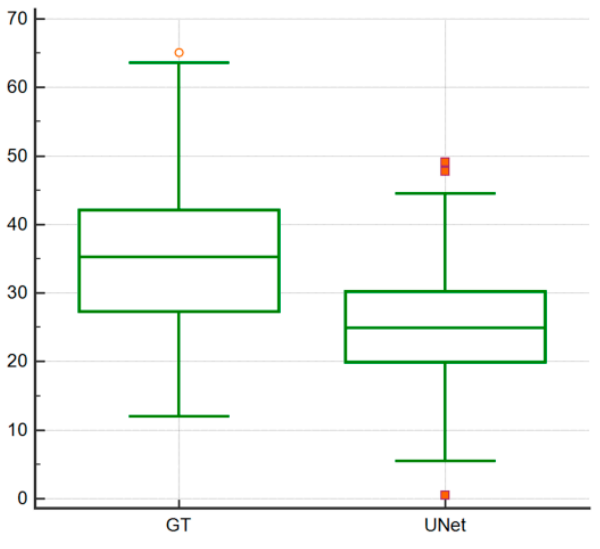

(a)

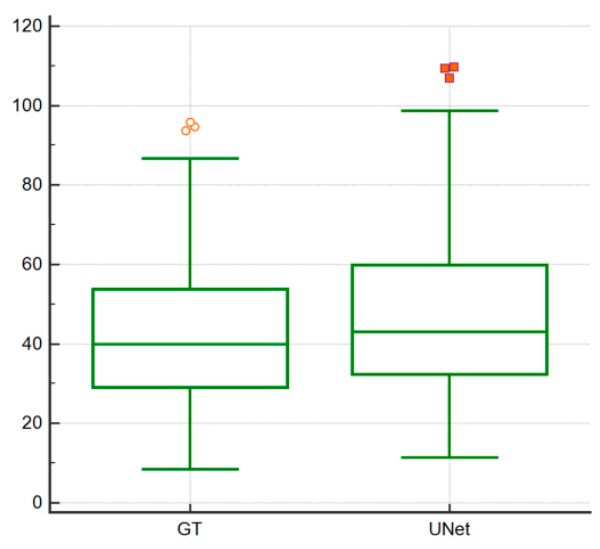

(b)

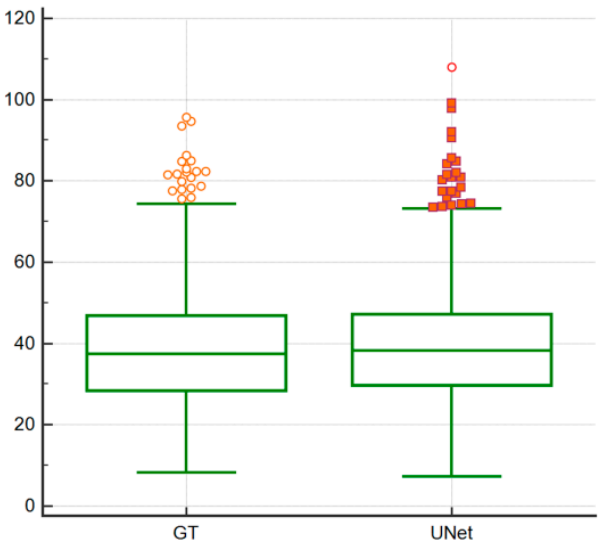

(c)

Figure 9. Comparison of paired $t$-test curves for Unseen AI and Seen AI database experiments. (a) Experiment \#1 (Unseen AI-1 (Tr: JAP, Te: HK)) (b) experiment \#2 (Unseen AI-2 (Tr: HK, Te: JAP)) (c) experiment \#3 (Seen AI-1 CV w/ Mixed). 


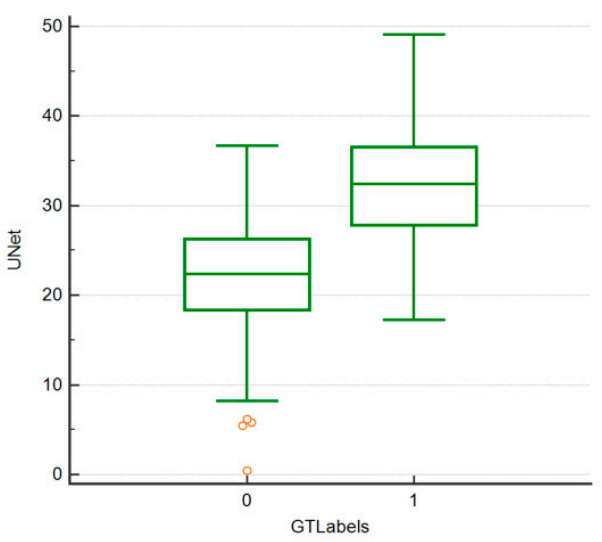

(a)

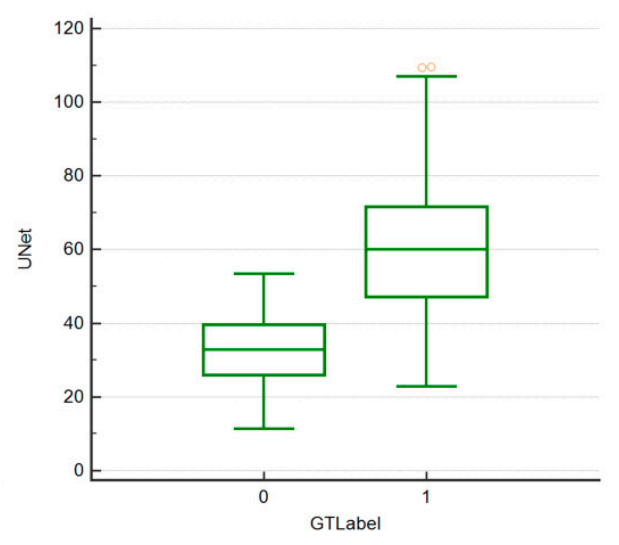

(b)

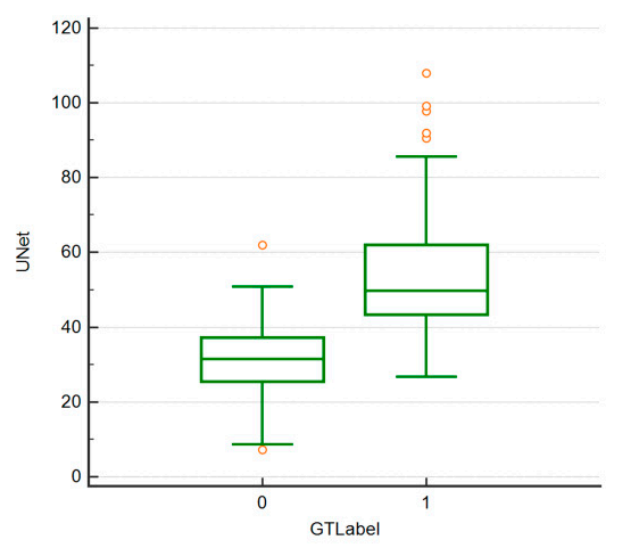

(c)

Figure 10. Comparison of ANOVA test curves for Unseen AI and Seen AI database experiments. (a) Experiment \#1 (Unseen AI-1 (Tr: JAP, Te: HK)) (b) experiment \#2 (Unseen AI-2 (Tr: HK, Te: JAP)) (c) experiment \#3 (Seen AI-1 CV w/ Mixed).

\subsection{Figure of Merit}

The figure of merit (FoM) is defined in terms of the central tendency of the error. Let $a_{a i}(n)$ and $a_{g t}$ represent the plaque area for the UNet model and GT, respectively, for image " $n$ ". Considering $N$ as the total number of scans, the corresponding mean area $\bar{a}_{a i}(e)$ for the experiment " $e$ " and for GT $\bar{a}_{g t}$ and then the FoM can be expressed as Equation (2):

$$
\operatorname{FoM}(e)=100-\left[\left(\frac{\left|\bar{a}_{a i}(e)-\bar{a}_{g t}\right|}{\bar{a}_{g t}}\right) * 100\right]
$$

Thus, the FoM for experiment \#1 (Unseen AI-1 (Tr: JAP, Te: HK)), experiment \#2 (Unseen AI-2 (Tr: HK, Te: JAP)), experiment \#3 (Seen AI-1 CV w/ Mixed), experiment \#4 (Seen AI-2 CV w / JAP), and experiment \#5 (Seen AI-3 CV w/ HK) was calculated using the above method to be $70.96 \%, 91.14 \%, 97.57 \%, 88.89 \%$, and $99.14 \%$, respectively.

\section{Discussion}

We presented herein the Unseen AI-based deep learning system for the segmentation of carotid B-mode plaque images. The system used two ethnic databases, i.e., the low-plaque Japanese diabetic database and the Hong Kong database. Furthermore, we hypothesized that the system is able to perform well with Unseen AI data. To prove our hypothesis, we performed a series of experiments as shown in Table 1. One unseen experiment used one ethnic DB for training and the other used another ethnic DB for testing and the second experiment did the opposite. The third experiment used a mix of the Japanese and Hong Kong databases for training and testing. In this Seen AI experiment, a cross-validation approach was used to test each image from the mixed database. However, in this mixed data experiment, the training and testing images were from the same pool of the mixed database. We also extended our work to experiments \#4 and \#5, which performed the cross-validation of same ethnic databases on the Japanese and Hong Kong databases. A comparison of the ROC curves for experiments \#1,2, and 3 is shown in Figure 11. Comparing the three seen and two unseen experiments, we can conclude that the system can segment a low atherosclerotic plaque from the unseen database. Additionally, the differences between unseen and seen experiments shown in Table 4 are within the range of $15 \%$. 


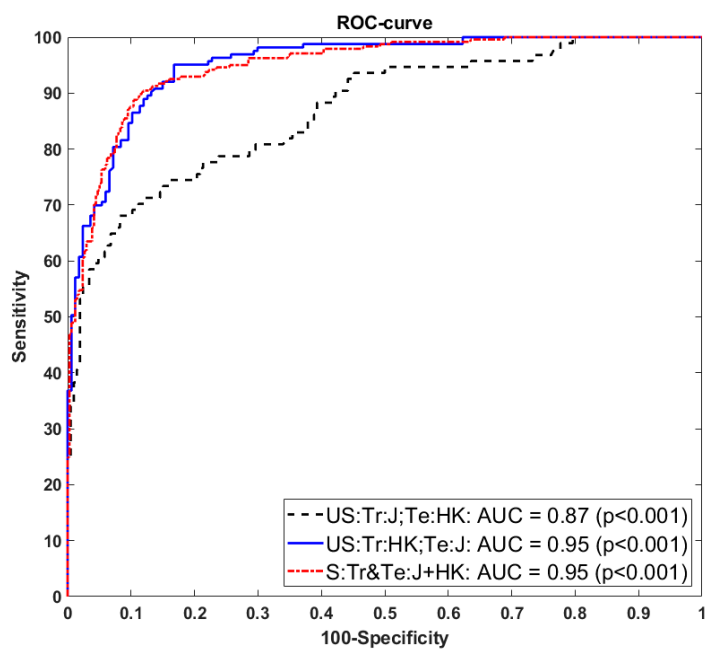

Figure 11. Comparison of ROC curve for unseen and seen database experiments.

Table 4. Comparison of experiments and validation of hypothesis.

\begin{tabular}{|c|c|c|c|c|c|c|c|c|c|c|}
\hline \#Exp & Comparison of Experiments & $\mathrm{CC}$ & AUC & $\mathrm{ACC}$ & Sens & Spec & Prec & MCC & DSC & JI \\
\hline \multirow[t]{2}{*}{$3-1$} & $\begin{array}{c}\text { Seen AI-1 CV w/ Mixed-Unseen AI-1 (Tr: JAP, } \\
\text { Te: HK) }\end{array}$ & 13.04 & 8.42 & 0.46 & -10.47 & 0.88 & 23.41 & 8.53 & 9.79 & 15.41 \\
\hline & & $\sim$ & $\sqrt{ }$ & $\sqrt{ }$ & $\sqrt{ }$ & $\sqrt{ }$ & $\sim$ & $\sqrt{ }$ & $\sqrt{ }$ & $\sim$ \\
\hline \multirow[t]{2}{*}{$3-2$} & $\begin{array}{c}\text { Seen AI-1 CV w/ Mixed-Unseen AI-2 (Tr: HK, } \\
\text { Te: JAP) }\end{array}$ & 5.43 & 1.05 & 0.34 & 7.93 & 0.05 & 1.42 & 5.06 & 5.06 & 8.22 \\
\hline & & $\sqrt{ }$ & $\sqrt{ }$ & $\sqrt{ }$ & $\sqrt{ }$ & $\sqrt{ }$ & $\sqrt{ }$ & $\sqrt{ }$ & $\sqrt{ }$ & $\sqrt{ }$ \\
\hline \multirow[t]{2}{*}{ 4-1 } & $\begin{array}{c}\text { Seen AI-2, CV w/ JAP-Unseen AI-1 (Tr: JAP, } \\
\text { Te: HK) }\end{array}$ & 8.05 & 6.45 & 0.44 & -4.56 & 0.62 & 16.28 & 6.59 & 7.41 & 12.33 \\
\hline & & $\sqrt{ }$ & $\sqrt{ }$ & $\sqrt{ }$ & $\sqrt{ }$ & $\sqrt{ }$ & $\sim$ & $\sqrt{ }$ & $\sqrt{ }$ & $\sim$ \\
\hline \multirow[t]{2}{*}{$4-2$} & $\begin{array}{c}\text { Seen AI-2, CV w/ JAP-Unseen AI-2 (Tr: HK, } \\
\text { Te: JAP) }\end{array}$ & 0.00 & -1.08 & 0.32 & 12.85 & -0.21 & -7.75 & 3.05 & 2.55 & 4.88 \\
\hline & & $\sqrt{ }$ & $\sqrt{ }$ & $\sqrt{ }$ & $\sim$ & $\sqrt{ }$ & $\sqrt{ }$ & $\sqrt{ }$ & $\sqrt{ }$ & $\sqrt{ }$ \\
\hline \multirow[t]{2}{*}{$5-1$} & $\begin{array}{c}\text { Seen AI-3, CV w/ H -Unseen AI-1 (Tr: JAP, Te: } \\
\text { HK) }\end{array}$ & 10.11 & 8.42 & 0.41 & -9.33 & 0.79 & 21.60 & 7.85 & 9.17 & 14.58 \\
\hline & & $\sqrt{ }$ & $\sqrt{ }$ & $\sqrt{ }$ & $\sqrt{ }$ & $\sqrt{ }$ & $\sim$ & $\sqrt{ }$ & $\sqrt{ }$ & $\sim$ \\
\hline \multirow[t]{2}{*}{$5-2$} & $\begin{array}{c}\text { Seen AI-3, CV w/ H -Unseen AI-2 (Tr: HK, Te: } \\
\text { JAP) }\end{array}$ & 2.25 & 1.05 & 0.29 & 8.88 & -0.04 & -0.91 & 4.36 & 4.40 & 7.32 \\
\hline & & $\sqrt{ }$ & $\sqrt{ }$ & $\sqrt{ }$ & $\sqrt{ }$ & $\sqrt{ }$ & $\sqrt{ }$ & $\sqrt{ }$ & $\sqrt{ }$ & $\sqrt{ }$ \\
\hline \multirow[t]{2}{*}{$3-4$} & $\begin{array}{l}\text { Seen AI-1 CV w/ Mixed-Seen AI-2, CV w/ } \\
\text { JAP }\end{array}$ & 5.43 & 2.11 & 0.02 & -5.65 & 0.26 & 8.51 & 2.08 & 2.58 & 3.52 \\
\hline & & $\sqrt{ }$ & $\sqrt{ }$ & $\sqrt{ }$ & $\sqrt{ }$ & $\sqrt{ }$ & $\sqrt{ }$ & $\sqrt{ }$ & $\sqrt{ }$ & $\sqrt{ }$ \\
\hline \multirow[t]{2}{*}{$3-5$} & $\begin{array}{c}\text { Seen AI-1 CV w / Mixed-Seen AI-3, CV w / } \\
\text { HK }\end{array}$ & 3.26 & 0.00 & 0.05 & -1.04 & 0.09 & 2.32 & 0.74 & 0.69 & 0.97 \\
\hline & & $\sqrt{ }$ & $\sqrt{ }$ & $\sqrt{ }$ & $\sqrt{ }$ & $\sqrt{ }$ & $\sqrt{ }$ & $\sqrt{ }$ & $\sqrt{ }$ & $\sqrt{ }$ \\
\hline
\end{tabular}

\subsection{Benchmarking}

Table 5 shows the benchmarking studies that involve the multi-ethnic databases for atherosclerotic plaque measurement. Modern techniques for the segmentation of the wall plaque in carotid B-mode ultrasound have been active since 2010. Even though the ultimate goal of these techniques is cIMT measurement, we briefly discuss them here. Most of the methods were developed by Suri's group from AtheroPoint ${ }^{\mathrm{TM}}$ (Roseville, CA, USA) [52,53]. 
In the early start-up methods for wall segmentation, the first-order absolute moment (FOAM) method for completely automated local statistical-based method (CALSFORM) was shown [54-56]. The region of interest detection was important for automated LI/MA detection. Three methods were discussed for ROI estimation in which the plaque wall was supposed to be detected [57]. Scale-space methods were invented, which had the flexibility of automatically finding the LI and MA borders, which proved to be more robust $[29,30]$. These techniques are also summarized in the review by Molinari et al. [58]. This system was then commercialized into AtheroEdge ${ }^{\mathrm{TM}} 1.0$ and applied for a multicenter clinical study. A review was published comparing the different methods [59]. Several validation methods and applications were also developed $[60,61]$. All the above studies were focused on wall segmentation and cIMT measurement. Recently, AI tools were developed for the joint estimation of wall thickness and area estimation by Biswas et al. [10]. Molinari et al. used five ethnic databases for IMT measurement using automated and semi-automated methods [15]. However, their systems did not involve deep-learning methods. Mean $\pm \mathrm{SD}$ for IMT measurement for all these methods were $0.811 \pm 0.292$ (CALEX), $0.779 \pm 0.264$ (CARES), $0.806 \pm 0.294$ (CAMES), $0.873 \pm 0.323$ (CAUDLES-EF), and $0.786 \pm 0.251$ (FOAM), respectively (in $\mathrm{mm}$ ). Ikeda et al. used three ethnic groups' (Japanese, Italy and Hong Kong) data for IMT measurement at the bulb area [62]. Another group of Zhou et al. [47] presented a deep learning-based method for segmentation of atherosclerotic plaque from carotid ultrasound images. Their system used two ethnic databases: namely those of Stroke Prevention and Atherosclerosis Research Centre (SPARC), from London, Canada; and Chinese data from Zhongnan Hospital (Wuhan, China). However, their work does not focus on the effect of unseen databases on the system's performance. In a multi-ethnic atherosclerosis study, Carol et al. 2018 used four ethnic people, i.e., White, Chinese, Black, and Hispanic people from across the country [63]. Purpose of their study was to analyze the relation between TPA and CVD risk factors in middle age group. Recently, by the team of Suri, Jamthikar et al. in 2020 proposed a Framingham risk score-based model for stroke risk assessment. They included 648 patients from three ethnic databases, and both left and right carotid artery images of each patient were included in the experiment. A 10-year risk prediction model, atherosclerosis CVD (ASCVD) developed by American College of Cardiology/American Heart Association, was used in their research. Furthermore, we compared our system with these studies, and found that our study has been solely focused upon the effect of ethnicity on the performance of the system [64].

Table 5. Benchmarking table showing multiethnic database studies for atherosclerotic plaque measurement.

\begin{tabular}{|c|c|c|c|c|c|}
\hline Sr\# & Authors and Year & Cohorts & Images & Purpose & Model \\
\hline 1 & Molinari et al., 2012 [15] & $\begin{array}{c}\text { Torino (n1) } \\
\text { Nicosia (n2) } \\
\text { Cagliari (n3) } \\
\text { Porto (n4) } \\
\text { Hong Kong (n5) }\end{array}$ & $\begin{array}{c}\mathrm{n} 1=200 \\
\mathrm{n} 2=100 \\
\mathrm{n} 3=42 \\
\mathrm{n} 4=23 \\
\mathrm{n} 5=300\end{array}$ & $\begin{array}{l}\text { IMT measurement using } \\
\text { auto and semi-auto } \\
\text { methods }\end{array}$ & ML \\
\hline 2 & Ikeda et al., 2013 [62] & $\begin{array}{l}\text { Japanese (n1) } \\
\text { Italy (n2) } \\
\text { Hong Kong (n3) }\end{array}$ & $\begin{array}{c}\mathrm{n} 1=259 \\
\mathrm{n} 2=98 \\
\mathrm{n} 3=300\end{array}$ & $\begin{array}{l}\text { IMT measurement in } \\
\text { Bulb area }\end{array}$ & ML \\
\hline 3 & Zhou et al., 2020 [47] & $\begin{array}{c}\text { SPARC (n1) } \\
\text { Chinese * (n2) }^{*}\end{array}$ & $\begin{array}{l}\mathrm{n} 1=510 \\
\mathrm{n} 2=638\end{array}$ & $\begin{array}{c}\text { Plaque area } \\
\text { measurement in ICA } \\
\text { and CCA images }\end{array}$ & DL \\
\hline 4 & Carol et al., 2018 [63] & $\begin{array}{l}\text { White (n1) } \\
\text { Chinese (n2) } \\
\text { Black (n3) } \\
\text { Hispanic (n4) }\end{array}$ & $\begin{array}{l}\mathrm{n} 1=946 \\
\mathrm{n} 2=185 \\
\mathrm{n} 3=595 \\
\mathrm{n} 4=479\end{array}$ & $\begin{array}{l}\text { Carotid plaque analysis } \\
\text { using manual method }\end{array}$ & Statistical method \\
\hline
\end{tabular}


Table 5. Cont.

\begin{tabular}{cccccc}
\hline Sr\# & Authors and Year & Cohorts & Images & Purpose & Model \\
\hline \multirow{2}{*}{5} & Jamathikar et al., 2020 [64] & Japanese (n1) & Asian-Indian (n2) & $\mathrm{n} 1=404$ \\
Spanish $(\mathrm{n} 3)$ & $\mathrm{n} 2=628$ & $\begin{array}{c}\text { Framingham risk } \\
\text { score-based stroke risk } \\
\text { stratification }\end{array}$ & ML \\
\hline \multirow{2}{*}{6} & Proposed method & Japanese (n1) & $\mathrm{n} 1=330$ & $\begin{array}{c}\text { Plaque area } \\
\text { measurement in CCA } \\
\text { images }\end{array}$ & DL \\
\hline
\end{tabular}

SPARC: Stroke Prevention and Atherosclerosis Research Centre, London, Canada; ${ }^{*}$ Zhongnan Hospital (Wuhan, China).

\subsection{Short Note on Image Quality}

Image quality in medical imaging such as MRI [65], CT [66], X-ray [67], and US [68] certainly plays an important point during the design of the computer-aided diagnosis (CAD) system. This is even more crucial during the US CAD design, since the quality of the images are subjected to several factors such as (i) strong contact between the probe and the skin; (ii) the gel used at the contact point; (iii) the gain control systems of the scanner; and (iv) the role of the harmonic imaging system and compound imaging systems were on during the beam formation and scanning process.

There have been several CAD systems which directly employ denoising solutions for US scans [13,69] or scale-space filtering methods [70]. One way to handle the denoising process is to compute the signal-to-noise ratio (SNR) or contrast-to-noise ratio (CNR) of US scans [71] and if they are under threshold limits, they can then be used for deep learning solutions. Note that these threshold limits are partially dependent on the datasets. Not all datasets need denoising solutions. Lastly, the latest US scanners have started to produce high-resolution images. Old legacy machines certainly need special denoisers for removing salt and pepper noise, speckle noise, or even getting rid of black shadows.

In our study, the images were selected by the experienced sonographer and filtered out based on their judgement of image quality to characterize the plaque area. Thus, we never encountered images which had low image quality. One can, however, design a method which can add denoisers in the loss function for improvements in the plaque detection process. This is similar to the approach of adding the penalty function in the boundary segmentation models [72] or using a partial differential equation-based smoothing process [73].

In our current study, we used two types of databases, namely the Japanese and Hong Kong ones. The Hong Kong database images are much nosier compared to the Japanese database images. The major objective of this paper was to address the use of different databases and prepare such a deep learning model which can train on one database and predict on other databases. We also swapped the databases and each time the accuracy was above the threshold mark. However, the images which were low in quality or the sonographer's lack of experience were not considered in our study.

\subsection{Strength, Limitations and Future Extensions}

As seen in Table 4, the percentage difference between the seen and unseen data experiments suggests that the system can perform atherosclerotic plaque segmentation. The system demonstrated significant results with unseen data experiments. Additionally, the system is able to capture the plaque data in the low-contrast images of the Hong Kong ethnic database. Furthermore, the system performs the testing of images in almost realtime. A major limitation of our system was the inclusion of the noisy Hong Kong database in the system. However, with the inclusion of some noise suppression methods [69], performance can be improved. Furthermore, (i) the system can be extended and applied for the segmentation of temporal data and (ii) we can characterize the segmented plaque wall using classification methods [74]. Multi-modality validation can also be achieved using joint ultrasound and CT [75]. 
The loss function plays an important role during the segmentation and classification paradigm [46]. In our recent publication, we tried to apply the dice loss function to stroke publications $[37,76]$. This was attempted for heavy plaque images having a partial volume effect (partial plaque available in the plaque zones of some pixel locations). The results were very encouraging. In our current study, most of the arteries were low plaque and mainly straight due to the nature of the common carotid artery. Thus, the identification of the region-of-interest was very straight-forward in the patient test data. There is no such issue of partial volume because the media region clearly showed a high-intensity zone. Thus, the simple cross-entropy function was successful in demonstrating our hypothesis of "Unseen AI". However, we will pursue other loss functions in the future such as MSE and DSC loss functions.

\section{Conclusions}

This was the first pilot study in the area of carotid ultrasound-based imaging that used two different ethnic groups in the AI framework, demonstrating the concept of "Unseen $\mathrm{AI}^{\prime \prime}$. Two distinct cohorts, i.e., the Japanese and Hong Kong ones, were used to prove

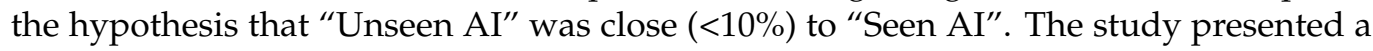
four-layer deep learning UNet architecture for atherosclerotic plaque wall segmentation in the common carotid arteries. Our performance parameters such as mean accuracy, dicesimilarity, and correlation-coefficient were 98.55, 78.38, and $0.80(p<0.0001)$, respectively, when using the Unseen AI pair-1 with the Japanese database for training and the Hong Kong database for testing. The same parameters were 98.67, 82.49, and $0.87(p<0.0001)$, respectively, when using the Unseen AI pair-2 consisting of Hong Kong training and Japanese testing. When benchmarking against the Seen AI, using the cross-validation protocol, for a mixed cohort (Japanese and Hong Kong), our system demonstrated the same parameters to be $99.01,86.89$, and $0.92(p<0.0001)$, respectively, validating our hypothesis and stability of the system. We further concluded that an online system takes less than one second, and such a system can be extended to other deep learning and hybrid deep learning models.

Author Contributions: Conceptualization, P.K.J. and J.S.S.; Methodology and software, P.K.J.; Validation, L.S., K.I.P. and A.J.; Investigation, M.K.K., J.R.L.; Resources, J.S.S., N.S.; Data curation, A.N.N., J.S.S.; Writing—original draft preparation, P.K.J., J.S.S.; Writing—review and editing, P.K.J., J.S.S.; Visualization, P.K.J.; Supervision, M.K.K., A.N.N., N.S.; Project administration, N.S. and J.S.S. All authors have read and agreed to the published version of the manuscript.

Funding: This research received no external funding.

Institutional Review Board Statement: Ethics approval was granted by the institutional review board (IRB) of Ohashi Ethics Committee, Toho University, Japan. Approval number Hashijo 13-56, 31 October 2013.

Informed Consent Statement: All patients were informed and consent was obtained before their inclusion in study.

Data Availability Statement: The Institutional Review Board has been issued to AtheroPoint, Roseville, CA, USA and therefore this database cannot be shared publically.

Conflicts of Interest: The authors declare no conflict of interest.

\section{References}

1. Centers of Disease Control and Prevention. U.S. Department of Health \& Human Services; Centers of Disease Control and Prevention: Atlanta, GA, USA, 2018.

2. Suri, J.S.; Kathuria, C.; Molinari, F. Atherosclerosis Disease Management; Springer Inc.: New York, NY, USA, $2010 ;$ p. 944.

3. Libby, P.; Buring, J.; Badimon, L.; Hansson, G.; Deanfield, J.; Bittencourt, M.; Tokgözoğlu, L.; Lewis, E.F. Atherosclerosis. Nat. Rev. Dis. Primers 2019, 5, 56. [CrossRef]

4. Patel, A.; Suri, H.S.; Singh, J.; Kumar, D.; Shafique, S.; Nicolaides, A.; Jain, S.K.; Saba, L.; Gupta, A.; Laird, J.R.; et al. A Review on Atherosclerotic Biology, Wall Stiffness, Physics of Elasticity, and Its Ultrasound-Based Measurement. Curr. Atheroscler. Rep. 2016, 18, 83. [CrossRef] 
5. Viswanathan, V.; Jamthikar, A.D.; Gupta, D.; Shanu, N.; Puvvula, A.; Khanna, N.N.; Saba, L.; Omerzum, T.; Viskovic, K.; Mavrogeni, S.; et al. Low-Cost Preventive Screening Using Carotid Ultrasound in Patients with Diabetes. Front. Biosci. 2020, 25, 1132-1171.

6. Porcu, M.; Mannelli, L.; Melis, M.; Suri, J.S.; Gerosa, C.; Cerrone, G.; DeFazio, G.; Faa, G.; Saba, L. Carotid Plaque Imaging Profiling in Subjects with Risk Factors (Diabetes and Hypertension). Cardiovasc. Diagn. Ther. 2020, 10, 1005-1018. [CrossRef]

7. Jamthikar, A.D.; Puvvula, A.; Gupta, D.; Johri, A.M.; Nambi, V.; Khanna, N.N.; Saba, L.; Mavrogeni, S.; Laird, J.R.; Pareek, G.; et al. Cardiovascular Disease and Stroke Risk Assessment in Patients with Chronic Kidney Disease Using Integration of Estimated Glomerular Filtration Rate, Ultrasonic Image Phenotypes, and Artificial Intelligence: A Narrative Review. Int. Angiol. 2020, 40, 150-164. [CrossRef]

8. Corrias, G.; Cocco, D.; Suri, J.S.; Meloni, L.; Cademartiri, F.; Saba, L. Heart Applications of 4d Flow. Cardiovasc. Diagn. Ther. 2020, 10, 1140-1149. [CrossRef]

9. Suri, J.S.; Laxminarayan, S. Angiography and Plaque Imaging: Advanced Segmentation Techniques; CRC Press: Boca Raton, FL, USA, 2003.

10. Biswas, M.; Saba, L.; Chakrabartty, S.; Khanna, N.N.; Song, H.; Suri, H.S.; Sfikakis, P.P.; Mavrogeni, S.; Viskovic, K.; Laird, J.R.; et al. Two-Stage Artificial Intelligence Model for Jointly Measurement of Atherosclerotic Wall Thickness and Plaque Burden in Carotid Ultrasound: A Screening Tool for Cardiovascular/Stroke Risk Assessment. Comput. Biol. Med. 2020, $123,103847$. [CrossRef] [PubMed]

11. Liu, K.; Suri, J.S. Automatic Vessel Indentification for Angiographic Screening. U.S. Patent 6845260B2, 18 January 2005.

12. Saba, L.; Suri, J.S. Multi-Detector Ct Imaging: Principles, Head, Neck, and Vascular Systems; CRC Press: Boca Raton, FL, USA, 2013; Volume 1.

13. Seabra, J.; Sanches, J. Ultrasound Imaging: Advances and Applications; Springer: New York, NY, USA, 2012.

14. Suri, J.S. Advances in Diagnostic and Therapeutic Ultrasound Imaging; Artech House: London, UK, 2008.

15. Molinari, F.; Meiburger, K.M.; Saba, L.; Acharya, U.R.; Ledda, G.; Zeng, G.; Ho, S.Y.S.; Ahuja, A.T.; Ho, S.C.; Nicolaides, A.; et al. Ultrasound IMT Measurement on a Multi-Ethnic and Multi-Institutional Database: Our Review and Experience Using Four Fully Automated and One Semi-Automated Methods. Comput. Methods Programs Biomed. 2012, 108, 946-960. [CrossRef] [PubMed]

16. Saba, L.; Meiburger, K.M.; Molinari, F.; Ledda, G.; Anzidei, M.; Acharya, U.R.; Zeng, G.; Shafique, S.; Nicolaides, A.; Suri, J.S. Carotid Imt Variability (Imtv) and Its Validation in Symptomatic Versus Asymptomatic Italian Population: Can This Be a Useful Index for Studying Symptomaticity? Echocardiography 2012, 29, 1111-1119. [CrossRef]

17. Lucatelli, P.; Raz, E.; Saba, L.; Argiolas, G.M.; Montisci, R.; Wintermark, M.; King, K.S.; Molinari, F.; Ikeda, N.; Siotto, P.; et al. Relationship between Leukoaraiosis, Carotid Intima-Media Thickness and Intima-Media Thickness Variability: Preliminary Results. Eur. Radiol. 2016, 26, 4423-4431. [CrossRef] [PubMed]

18. Johri, A.M.; Lajkosz, K.A.; Grubic, N.; Islam, S.; Li, T.Y.; Simpson, C.S.; Ewart, P.; Suri, J.S.; Hétu, M.-F. Maximum Plaque Height in Carotid Ultrasound Predicts Cardiovascular Disease Outcomes: A Population-Based Validation Study of the American Society of Echocardiography's Grade II-III Plaque Characterization and Protocol. Int. J. Cardiovasc. Imaging 2021, 37, 1601-1610. [CrossRef] [PubMed]

19. Puvvula, A.; Jamthikar, A.D.; Gupta, D.; Khanna, N.N.; Porcu, M.; Saba, L.; Viskovic, K.; Ajuluchukwu, J.N.A.; Gupta, A.; Mavrogeni, S.; et al. Morphological Carotid Plaque Area Is Associated with Glomerular Filtration Rate: A Study of South Asian Indian Patients With Diabetes and Chronic Kidney Disease. Angiology 2020, 71, 520-535. [CrossRef]

20. Kotsis, V.; Jamthikar, A.D.; Araki, T.; Gupta, D.; Laird, J.R.; Giannopoulos, A.A.; Saba, L.; Suri, H.S.; Mavrogeni, S.; Kitas, G.D.; et al. Echolucency-Based Phenotype in Carotid Atherosclerosis Disease for Risk Stratification of Diabetes Patients. Diabetes Res. Clin. Pract. 2018, 143, 322-331. [CrossRef]

21. Cuadrado-Godia, E.; Maniruzzaman; Araki, T.; Puvvula, A.; Rahman, J.; Saba, L.; Suri, H.S.; Gupta, A.; Banchhor, S.K.; Teji, J.S.; et al. Morphologic TPA (mTPA) and Composite Risk Score for Moderate Carotid Atherosclerotic Plaque Is Strongly Associated with Hba1c in Diabetes Cohort. Comput. Biol. Med. 2018, 101, 128-145. [CrossRef]

22. Kumar, P.K.; Araki, T.; Rajan, J.; Laird, J.R.; Nicolaides, A.; Suri, J.S. State-of-the-Art Review on Automated Lumen and Adventitial Border Delineation and Its Measurements in Carotid Ultrasound. Comput. Methods Programs Biomed. 2018, 163, 155-168. [CrossRef] [PubMed]

23. Kumar, P.K.; Araki, T.; Rajan, J.; Saba, L.; Lavra, F.; Ikeda, N.; Sharma, A.M.; Shafique, S.; Nicolaides, A.; Laird, J.R.; et al. Accurate Lumen Diameter Measurement in Curved Vessels in Carotid Ultrasound: An Iterative Scale-Space and Spatial Transformation Approach. Med. Biol. Eng. Comput. 2017, 55, 1415-1434. [CrossRef] [PubMed]

24. Saba, L.; Banchhor, S.K.; Londhe, N.D.; Araki, T.; Laird, J.R.; Gupta, A.; Nicolaides, A.; Suri, J.S. Web-Based Accurate Measurements of Carotid Lumen Diameter and Stenosis Severity: An Ultrasound-Based Clinical Tool for Stroke Risk Assessment During Multicenter Clinical Trials. Comput. Biol. Med. 2017, 91, 306-317. [CrossRef]

25. Saba, L.; Jamthikar, A.; Gupta, D.; Khanna, N.N.; Viskovic, K.; Suri, H.S.; Gupta, A.; Mavrogeni, S.; Turk, M.; Laird, J.R.; et al. Global Perspective on Carotid Intima-Media Thickness and Plaque: Should the Current Measurement Guidelines Be Revisited? Int. Angiol. 2020, 38, 451-465. [CrossRef]

26. Saba, L.; Agarwal, N.; Cau, R.; Gerosa, C.; Sanfilippo, R.; Porcu, M.; Montisci, R.; Cerrone, G.; Qi, Y.; Balestrieri, A.; et al. Review of Imaging Biomarkers for the Vulnerable Carotid Plaque. JVS Vasc. Sci. 2021, 2, 149-158. [CrossRef] 
27. Saba, L.; Banchhor, S.K.; Araki, T.; Viskovic, K.; Londhe, N.D.; Laird, J.R.; Suri, H.S.; Suri, J.S. Intra- and Inter-Operator Reproducibility of Automated Cloud-Based Carotid Lumen Diameter Ultrasound Measurement. Indian Heart J. 2018, 70, 649-664. [CrossRef]

28. Saba, L.; Banchhor, S.K.; Araki, T.; Suri, H.S.; Londhe, N.D.; Laird, J.R.; Višković, K.; Suri, J.S. Intra- and Inter-Operator Reproducibility Analysis of Automated Cloud-based Carotid Intima Media Thickness Ultrasound Measurement. J. Clin. Diagn. Res. 2018, 12, 1-7. [CrossRef]

29. Molinari, F.; Zeng, G.; Suri, J.S. Intima-Media Thickness: Setting a Standard for a Completely Automated Method of Ultrasound Measurement. IEEE Trans. Ultrason. Ferroelectr. Freq. Control. 2010, 57, 1112-1124. [CrossRef]

30. Molinari, F.; Pattichis, C.S.; Zeng, G.; Saba, L.; Acharya, U.R.; Sanfilippo, R.; Nicolaides, A.; Suri, J.S. Completely Automated Multiresolution Edge Snapper-A New Technique for an Accurate Carotid Ultrasound Imt Measurement: Clinical Validation and Benchmarking on a Multi-Institutional Database. IEEE Trans. Image Process. 2012, 21, 1211-1222. [CrossRef]

31. Ayman El-Baz, J.S.S. Artificial Intelligence in Cancer Diagnosis and Prognosis. In Ipem-Iop Series in Physics and Engineering in Medicine and Biology; IOP Press: Bristol, UK, 2022; Volume 1.

32. Rangayyan, R.M.; Suri, J.S. Recent Advances in Breast Imaging, Mammography, and Computer-Aided Diagnosis of Breast Cancer; SPIE Publications: Bellingham, WA, USA, 2006.

33. Saba, L.; Jain, P.; Suri, H.S.; Ikeda, N.; Araki, T.; Singh, B.K.; Nicolaides, A.; Shafique, S.; Gupta, A.; Laird, J.R.; et al. Plaque Tissue Morphology-Based Stroke Risk Stratification Using Carotid Ultrasound: A Polling-Based Pca Learning Paradigm. J. Med. Syst. 2017, 41, 98. [CrossRef]

34. Araki, T.; Jain, P.; Suri, H.S.; Londhe, N.D.; Ikeda, N.; El-Baz, A.; Shrivastava, V.; Saba, L.; Nicolaides, A.; Shafique, S.; et al. Stroke Risk Stratification and Its Validation Using Ultrasonic Echolucent Carotid Wall Plaque Morphology: A Machine Learning Paradigm. Comput. Biol. Med. 2017, 80, 77-96. [CrossRef] [PubMed]

35. Maniruzzaman, M.; Rahman, J.; Hasan, A.-M.; Suri, H.S.; Abedin, M.; El-Baz, A.; Suri, J.S. Accurate Diabetes Risk Stratification Using Machine Learning: Role of Missing Value and Outliers. J. Med. Syst. 2018, 42, 92. [CrossRef]

36. Acharya, U.R.; Sree, S.V.; Krishnan, M.M.R.; Krishnananda, N.; Ranjan, S.; Umesh, P.; Suri, J.S. Automated Classification of Patients with Coronary Artery Disease Using Grayscale Features from Left Ventricle Echocardiographic Images. Comput. Methods Programs Biomed. 2013, 112, 624-632. [CrossRef] [PubMed]

37. Jain, P.K.; Sharma, N.; Giannopoulos, A.A.; Saba, L.; Nicolaides, A.; Suri, J.S. Hybrid Deep Learning Segmentation Models for Atherosclerotic Plaque in Internal Carotid Artery B-Mode Ultrasound. Comput. Biol. Med. 2021, 136, 104721. [CrossRef]

38. Biswas, M.; Kuppili, V.; Edla, D.R.; Suri, H.S.; Saba, L.; Marinhoe, R.T.; Sanches, J.; Suri, J.S. Symtosis: A Liver Ultrasound Tissue Characterization and Risk Stratification in Optimized Deep Learning Paradigm. Comput. Methods Programs Biomed. 2018, 155, 165-177. [CrossRef] [PubMed]

39. Jain, P.; Gupta, S.; Bhavsar, A.; Nigam, A.; Sharma, N. Localization of Common Carotid Artery Transverse Section in B-Mode Ultrasound Images Using Faster Rcnn: A Deep Learning Approach. Med. Biol. Eng. Comput. 2020, 58, 471-482. [CrossRef]

40. Saba, L.; Biswas, M.; Suri, H.S.; Viskovic, K.; Laird, J.R.; Cuadrado-Godia, E.; Nicolaides, A.; Khanna, N.N.; Viswanathan, V.; Suri, J.S. Ultrasound-Based Carotid Stenosis Measurement and Risk Stratification in Diabetic Cohort: A Deep Learning Paradigm. Cardiovasc. Diagn. Ther. 2019, 9, 439-461. [CrossRef] [PubMed]

41. Saba, L.; Sanagala, S.S.; Gupta, S.K.; Koppula, V.K.; Johri, A.M.; Sharma, A.M.; Kolluri, R.; Bhatt, D.L.; Nicolaides, A.; Suri, J.S. Ultrasound-Based Internal Carotid Artery Plaque Characterization Using Deep Learning Paradigm on a Supercomputer: A Cardiovascular Disease/Stroke Risk Assessment System. Int. J. Cardiovasc. Imaging 2021, 37, 1511-1528. [CrossRef] [PubMed]

42. Saba, L.; Sanagala, S.S.; Gupta, S.K.; Koppula, V.K.; Johri, A.M.; Khanna, N.N.; Mavrogeni, S.; Laird, J.R.; Pareek, G.; Miner, M.; et al. Multimodality Carotid Plaque Tissue Characterization and Classification in the Artificial Intelligence Paradigm: A Narrative Review for Stroke Application. Ann. Transl. Med. 2021, 9, 1206. [CrossRef] [PubMed]

43. Agarwal, M.; Saba, L.; Gupta, S.K.; Johri, A.M.; Khanna, N.N.; Mavrogeni, S.; Laird, J.R.; Pareek, G.; Miner, M.; Sfikakis, P.P.; et al. Wilson Disease Tissue Classification and Characterization Using Seven Artificial Intelligence Models Embedded with 3D Optimization Paradigm on a Weak Training Brain Magnetic Resonance Imaging Datasets: A Supercomputer Application. Med. Biol. Eng. Comput. 2021, 59, 511-533. [CrossRef]

44. Biswas, M.; Kuppili, V.; Saba, L.; Edla, D.R.; Suri, H.S.; Cuadrado-Godia, E.; Laird, J.R.; Marinhoe, R.T.; Sanches, J.M.; Nicolaides, A.; et al. State-of-the-Art Review on Deep Learning in Medical Imaging. Front. Biosci. 2019, 24, $392-426$.

45. Saba, L.; Biswas, M.; Kuppili, V.; Godia, E.C.; Suri, H.S.; Edla, D.R.; Omerzu, T.; Laird, J.R.; Khanna, N.N.; Mavrogeni, S.; et al. The Present and Future of Deep Learning in Radiology. Eur. J. Radiol. 2019, 114, 14-24. [CrossRef] [PubMed]

46. Jena, B.; Saxena, S.; Nayak, G.K.; Saba, L.; Sharma, N.; Suri, J.S. Artificial Intelligence-Based Hybrid Deep Learning Models for Image Classification: The First Narrative Review. Comput. Biol. Med. 2021, 137, 104803. [CrossRef]

47. Zhou, R.; Guo, F.; Azarpazhooh, M.R.; Hashemi, S.; Cheng, X.; Spence, J.D.; Ding, M.; Fenster, A. Deep Learning-Based Measurement of Total Plaque Area in B-Mode Ultrasound Images. IEEE J. Biomed. Health Inform. 2021, 25, 2967-2977. [CrossRef]

48. Suri, J.S.; Agarwal, S.; Gupta, S.K.; Puvvula, A.; Viskovic, K.; Suri, N.; Alizad, A.; El-Baz, A.; Saba, L.; Fatemi, M.; et al. Systematic Review of Artificial Intelligence in Acute Respiratory Distress Syndrome for COVID-19 Lung Patients: A Biomedical Imaging Perspective. IEEE J. Biomed. Health Inform. 2021, 25, 4128-4139. [CrossRef] 
49. Molinari, F.; Meiburger, K.M.; Saba, L.; Acharya, U.R.; Famiglietti, L.; Georgiou, N.; Nicolaides, A.; Mamidi, R.S.; Kuper, H.; Suri, J.S. Automated Carotid Imt Measurement and Its Validation in Low Contrast Ultrasound Database of 885 Patient Indian Population Epidemiological Study: Results of Atheroedge ${ }^{\circledR}$ Software. In Multi-Modality Atherosclerosis Imaging and Diagnosis; Springer: Berlin/Heidelberg, Germany, 2014; pp. 209-219.

50. Saba, L.; Banchhor, S.K.; Suri, H.S.; Londhe, N.D.; Araki, T.; Ikeda, N.; Viskovic, K.; Shafique, S.; Laird, J.R.; Gupta, A.; et al. Accurate Cloud-Based Smart IMT Measurement, Its Validation and Stroke Risk Stratification in Carotid Ultrasound: A Web-Based Point-of-Care Tool for Multicenter Clinical Trial. Comput. Biol. Med. 2016, 75, 217-234. [CrossRef]

51. Araki, T.; Aimbe, F.; Kumar, P.K.; Suri, H.S.; Ikeda, N.; Gupta, A.; Saba, L.; Rajan, J.; Lavra, F.; Sharma, A.M.; et al. Two Automated Techniques for Carotid Lumen Diameter Measurement: Regional Versus Boundary Approaches. J. Med. Syst. 2016, 40, 1-19. [CrossRef]

52. Molinari, F.; Liboni, W.; Giustetto, P.; Badalamenti, S.; Suri, J.S. Automatic Computer-Based Tracings (Act) in Longitudinal 2-D Ultrasound Images Using Different Scanners. J. Mech. Med. Biol. 2009, 9, 481-505. [CrossRef]

53. Molinari, F.; Zeng, G.; Suri, J.S. An Integrated Approach to Computer-Based Automated Tracing and Its Validation for 200 Common Carotid Arterial Wall Ultrasound Images: A New Technique. J. Ultrasound Med. 2010, 29, 399-418. [CrossRef]

54. Molinari, F.; Liboni, W.; Pantziaris, M.; Suri, J.S. CALSFOAM-Completed Automated Local Statistics Based First Order Absolute Moment for Carotid Wall Recognition, Segmentation and IMT Measurement: Validation and Benchmarking on a 300 Patient Database. Int. Angiol. 2011, 30, 227-241. [PubMed]

55. Meiburger, K.M.; Molinari, F.; Acharya, U.R.; Saba, L.; Rodrigues, P.; Liboni, W.; Nicolaides, A.; Suri, J.S. Automated Carotid Artery Intima Layer Regional Segmentation. Phys. Med. Biol. 2011, 56, 4073-4090. [CrossRef] [PubMed]

56. Molinari, F.; Meiburger, K.M.; Acharya, U.R.; Zeng, G.; Rodrigues, P.S.; Saba, L.; Nicolaides, A.; Suri, J.S. Cares 3.0: A Two Stage System Combining Feature-Based Recognition and Edge-Based Segmentation for CIMT Measurement on a Multi-Institutional Ultrasound Database of 300 Images. In Proceedings of the 2011 Annual International Conference of the IEEE Engineering in Medicine and Biology Society, Boston, MA, USA, 30 August-3 September 2011.

57. Molinari, F.; Meiburger, K.M.; Zeng, G.; Acharya, U.R.; Liboni, W.; Nicolaides, A.; Suri, J.S. Carotid Artery Recognition System: A Comparison of Three Automated Paradigms for Ultrasound Images. Med. Phys. 2011, 39, 378-391. [CrossRef] [PubMed]

58. Molinari, F.; Acharya, U.R.; Zeng, G.; Meiburger, K.M.; Suri, J.S. Completely Automated Robust Edge Snapper for Carotid Ultrasound IMT Measurement on a Multi-Institutional Database of 300 Images. Med. Biol. Eng. Comput. 2011, 49, 935-945. [CrossRef]

59. Molinari, F.; Zeng, G.; Suri, J.S. A State of the Art Review on Intima-Media Thickness (IMT) Measurement and Wall Segmentation Techniques for Carotid Ultrasound. Comput. Methods Programs Biomed. 2010, 100, 201-221. [CrossRef]

60. Saba, L.; Gao, H.; Raz, E.; Sree, S.V.; Mannelli, L.; Tallapally, N.; Molinari, F.; Bassareo, P.P.; Acharya, U.R.; Poppert, H.; et al. Semiautomated Analysis of Carotid Artery Wall Thickness in MRI. J. Magn. Reson. Imaging 2014, 39, 1457-1467. [CrossRef]

61. Ikeda, N.; Gupta, A.; Dey, N.; Bose, S.; Shafique, S.; Arak, T.; Godia, E.C.; Saba, L.; Laird, J.R.; Nicolaides, A.; et al. Improved Correlation between Carotid and Coronary Atherosclerosis Syntax Score Using Automated Ultrasound Carotid Bulb Plaque IMT Measurement. Ultrasound Med. Biol. 2015, 41, 1247-1262. [CrossRef]

62. Ikeda, N.; Dey, N.; Sharma, A.; Gupta, A.; Bose, S.; Acharjee, S.; Shafique, S.; Cuadrado-Godia, E.; Araki, T.; Saba, L.; et al. Automated Segmental-IMT Measurement in Thin/Thick Plaque with Bulb Presence in Carotid Ultrasound from Multiple Scanners: Stroke Risk Assessment. Comput. Methods Programs Biomed. 2017, 141, 73-81. [CrossRef] [PubMed]

63. Mitchell, C.; Korcarz, C.E.; Gepner, A.D.; Kaufman, J.D.; Post, W.; Tracy, R.; Gassett, A.J.; Ma, N.; McClelland, R.L.; Stein, J.H. Ultrasound Carotid Plaque Features, Cardiovascular Disease Risk Factors and Events: The Multi-Ethnic Study of Atherosclerosis. Atherosclerosis 2018, 276, 195-202. [CrossRef] [PubMed]

64. Jamthikar, A.; Gupta, D.; Cuadrado-Godia, E.; Puvvula, A.; Khanna, N.N.; Saba, L.; Viskovic, K.; Mavrogeni, S.; Turk, M.; Laird, J.R. Ultrasound-Based Stroke/Cardiovascular Risk Stratification Using Framingham Risk Score and Ascrd Risk Score Based on "Integrated Vascular Age" Instead of "Chronological Age": A Multi-Ethnic Study of Asian Indian, Caucasian, and Japanese Cohorts. Cardiovasc. Diagn. Ther. 2020, 10, 939. [CrossRef] [PubMed]

65. Kumar, P.K.; Darshan, P.; Kumar, S.; Ravindra, R.; Rajan, J.; Saba, L.; Suri, J.S. Magnetic Resonance Image Denoising Using Nonlocal Maximum Likelihood Paradigm in DCT-Framework. Int. J. Imaging Syst. Technol. 2015, 25, 256-264. [CrossRef]

66. Saba, L.; Di Martino, M.; Siotto, P.; Anzidei, M.; Argiolas, G.M.; Porcu, M.; Suri, J.S.; Wintermark, M. Radiation Dose and Image Quality of Computed Tomography of the Supra-Aortic Arteries: A Comparison between Single-Source and Dual-Source CT Scanners. J. Neuroradiol. 2018, 45, 136-141. [CrossRef] [PubMed]

67. Kumar, D.; Wei, L.; Turlapati, R.; Suri, J.S. Improvement in C-Arm Acquired DSA Image Quality via Combined Effect of Inverse Consistent Motion Correction and Nonlinear Normalization. In Proceedings of the 2007 29th Annual International Conference of the IEEE Engineering in Medicine and Biology Society, Lyon, France, 22-26 August 2007.

68. Suri, J.; Wu, D.; Gao, J.; Singh, S.; Laxminarayan, S. A Comparison of State-of-the-Art Diffusion Imaging Techniques for Smoothing Medical/Non-Medical Image Data. In Proceedings of the Object Recognition Supported by User Interaction for Service Robots, Quebec City, QC, Canada, 11-15 August 2002.

69. Sudeep, P.; Palanisamy, P.; Rajan, J.; Baradaran, H.; Saba, L.; Gupta, A.; Suri, J.S. Speckle Reduction in Medical Ultrasound Images Using an Unbiased Non-Local Means Method. Biomed. Signal Process. Control. 2016, 28, 1-8. [CrossRef] 
70. Suri, J.; Liu, K.; Reden, L.; Laxminarayan, S. White and Black Blood Volumetric Angiographic Filtering: Ellipsoidal Scale-Space Approach. IEEE Trans. Inf. Technol. Biomed. 2002, 6, 142-158. [CrossRef]

71. Banchhor, S.K.; Araki, T.; Londhe, N.D.; Ikeda, N.; Radeva, P.; Elbaz, A.; Saba, L.; Nicolaides, A.; Shafique, S.; Laird, J.R.; et al. Five Multiresolution-Based Calcium Volume Measurement Techniques from Coronary IVUS Videos: A Comparative Approach. Comput. Methods Programs Biomed. 2016, 134, 237-258. [CrossRef]

72. Suri, J.; Liu, K.; Singh, S.; Laxminarayan, S.; Zeng, X.; Reden, L. Shape Recovery Algorithms Using Level Sets in 2-D/3-D Medical Imagery: A State-of-the-Art Review. IEEE Trans. Inf. Technol. Biomed. 2002, 6, 8-28. [CrossRef]

73. Suri, J.S.; Laxminarayan, S. Pde and Level Sets; Springer Science \& Business Media: Berlin/Heidelberg, Germany, 2002.

74. Acharya, U.R.; Faust, O.; Sree, S.V.; Molinari, F.; Saba, L.; Nicolaides, A.; Suri, J.S. An Accurate and Generalized Approach to Plaque Characterization in 346 Carotid Ultrasound Scans. IEEE Trans. Instrum. Meas. 2012, 61, 1045-1053. [CrossRef]

75. Saba, L.; Tallapally, N.; Gao, H.; Molinari, F.; Anzidei, M.; Piga, M.; Sanfilippo, R.; Suri, J.S. Semiautomated and Automated Algorithms for Analysis of the Carotid Artery Wall on Computed Tomography and Sonography: A Correlation Study. J. Ultrasound Med. 2013, 32, 665-674. [CrossRef] [PubMed]

76. Biswas, M.; Kuppili, V.; Saba, L.; Edla, D.R.; Suri, H.S.; Sharma, A.; Cuadrado-Godia, E.; Laird, J.R.; Nicolaides, A.; Suri, J.S. Deep Learning Fully Convolution Network for Lumen Characterization in Diabetic Patients Using Carotid Ultrasound: A Tool for Stroke Risk. Med. Biol. Eng. Comput. 2019, 57, 543-564. [CrossRef] [PubMed] 